\title{
Exploitation des données LiDAR dans une approche géoarchéologique diachronique et multi-sources : l'exemple du plateau de Messigny sur le territoire du Val Suzon (Côte-d'Or).
}

\author{
Exploitation of LiDAR data in a diachronic and multi-source
} geoarchaeological approach: the example of the Messigny plateau on the Val Suzon territory (Côte-d'Or).

\author{
Rémi Landois ${ }^{1}$, Jean-Pierre Garcia ${ }^{2}$, Amélie Quiquerez ${ }^{3}$ \\ ${ }^{1}$ Doctorant à l'université de Bourgogne, UMR 6298 ARTEHIS, remi.landois@u-bourgogne.fr \\ ${ }^{2}$ Professeur à l'université de Bourgogne, UMR 6298 ARTEHIS, jean-pierre.garcia@u-bourgogne.fr \\ ${ }^{3}$ Maître de conférences à l'université de Bourgogne, UMR 6298 ARTEHIS, amelie.quiquerez@u-bourgogne.fr
}

RÉSUMÉ. Depuis 2015, la réserve naturelle régionale du Val Suzon (Côte-d'Or) fait l'objet de recherches archéologiques s'appuyant notamment sur l'analyse de données LiDAR acquises en 2013. Grâce à ces données, de nombreuses traces d'activités humaines préservées sous la forêt ont pu être identifiées et étudiées. Les structures archéologiques, se caractérisant par une grande diversité d'âges, d'origines et de natures, permettent ainsi d'appréhender l'évolution de ce territoire sur le temps long. Outre le LiDAR, diverses sources sont exploitées, comme les archives textuelles et les plans anciens. Ces documents apportent des informations complémentaires à celles issues du LiDAR et renseignent par exemple sur l'exploitation des ressources naturelles du Val Suzon ou encore sur la transformation du paysage au cours du temps. Cette approche diachronique et multi-sources est ici illustrée à travers l'étude de quelques structures repérées sur le plateau de Messigny. Du tumulus de l'âge du Bronze aux tranchées de la guerre de 1870, cette zone d'environ 200 hectares présente un large panel de structures archéologiques témoignant de l'histoire complexe de ce territoire aujourd'hui forestier. De plus, les conditions géomorphologiques de ce secteur ont permis la préservation exceptionnelle de traces laissées par le passage répété d'animaux. II apparaît cependant que ces traces, comme d'autres micro-reliefs, sont uniquement visibles dans les zones où la forêt s'est maintenue depuis plusieurs siècles, et ce malgré les épisodes de défrichements qu'a connus la commune de Messigny. Enfin, les recherches menées sur le Val Suzon abordent des problématiques propres à l'archéologie forestière ainsi qu'à l'histoire des forêts et à l'écologie historique, notamment en s'intéressant à la question de l'impact et des héritages des dynamiques passées sur l'environnement actuel.

ABSTRACT. Since 2015, the Regional Natural Reserve of Val Suzon (Côte-d'Or) has been the subject of archaeological researches based particularly on the analysis of LiDAR datas acquired in 2013. Thanks to these datas, many traces of activities preserved under the forest can be identified and studied. These archaeological structures, characterized by a great diversity of ages, origins and natures, make it possible to apprehend the evolution of this territory over the long term. In addition to LiDAR, various sources are also exploited, such as textual archives and old plans. These documents provide additional information to those from LiDAR and also provide information, for example, about the exploitation of Val Suzon's natural resources or the transformation of the landscape over time. This diachronic and multi-source approach is illustrated presently through the study of some structures identified on the plateau of Messigny. From the Bronze Age tumulus to the trenches of the war of 1870, this area of approximately 200 hectares presents a wide range of archaeological structures testifying to the composite history of this territory which is occupied nowadays by a forest. In addition, the geomorphological conditions of this area have allowed the preservation of exceptional traces left by the repeated passage of animals. It appears however that these traces, like other micro-reliefs, are only visible in the areas where the forest has been maintained for several centuries, despite the episodes of deforestation that occurred in Messigny. Finally, the research carried out on the Val Suzon addresses issues specific to forest archaeology as well as forest history and historical ecology, in particular by focusing on the question of the impact and legacies of past dynamics on the current environment.

MOTS-CLÉS. LiDAR, Forêt, Archéologie forestière, Bourgogne.

KEYWORDS. LiDAR, Forest, Forest archaeology, Burgundy. 
Le territoire du Val Suzon en Côte-d'Or a fait l'objet d'un levé LiDAR en 2013, dont les données sont depuis analysées dans le cadre de recherches archéologiques. Cette acquisition LiDAR couvre une large partie du bassin versant du Suzon, un affluent de l'Ouche, rivière qui traverse la ville de Dijon. Les recherches dont il est question dans le présent article sont centrées sur la réserve naturelle régionale du Val Suzon, qui correspond à un massif forestier de près de 3000 hectares réparti sur plusieurs communes (Étaules, Darois, Messigny-et-Vantoux et Val-Suzon) (Figure 1). Cette réserve rassemble une forêt domaniale et des bois communaux installés sur les plateaux calcaires et les versants qui bordent la vallée du Suzon. Outre le classement en réserve en 2011, le Val Suzon a obtenu le label «Forêt d'Exception ${ }^{\circledR} »$ décerné par l'Office national des forêts en octobre $2016^{1}$. Comme pour les massifs de Fontainebleau, Verdun ou Tronçais, ce label est décerné à des forêts emblématiques en raison notamment de leur patrimoine naturel et historique. Ainsi, le territoire du Val Suzon se caractérise par une mosaïque de milieux qui abritent une forte biodiversité. On y trouve également de nombreux vestiges archéologiques préservés par la forêt et révélés grâce au LiDAR. C'est pourquoi, en 2015, a démarré un projet de recherches scientifiques sur l'histoire de ce territoire ${ }^{2}$.

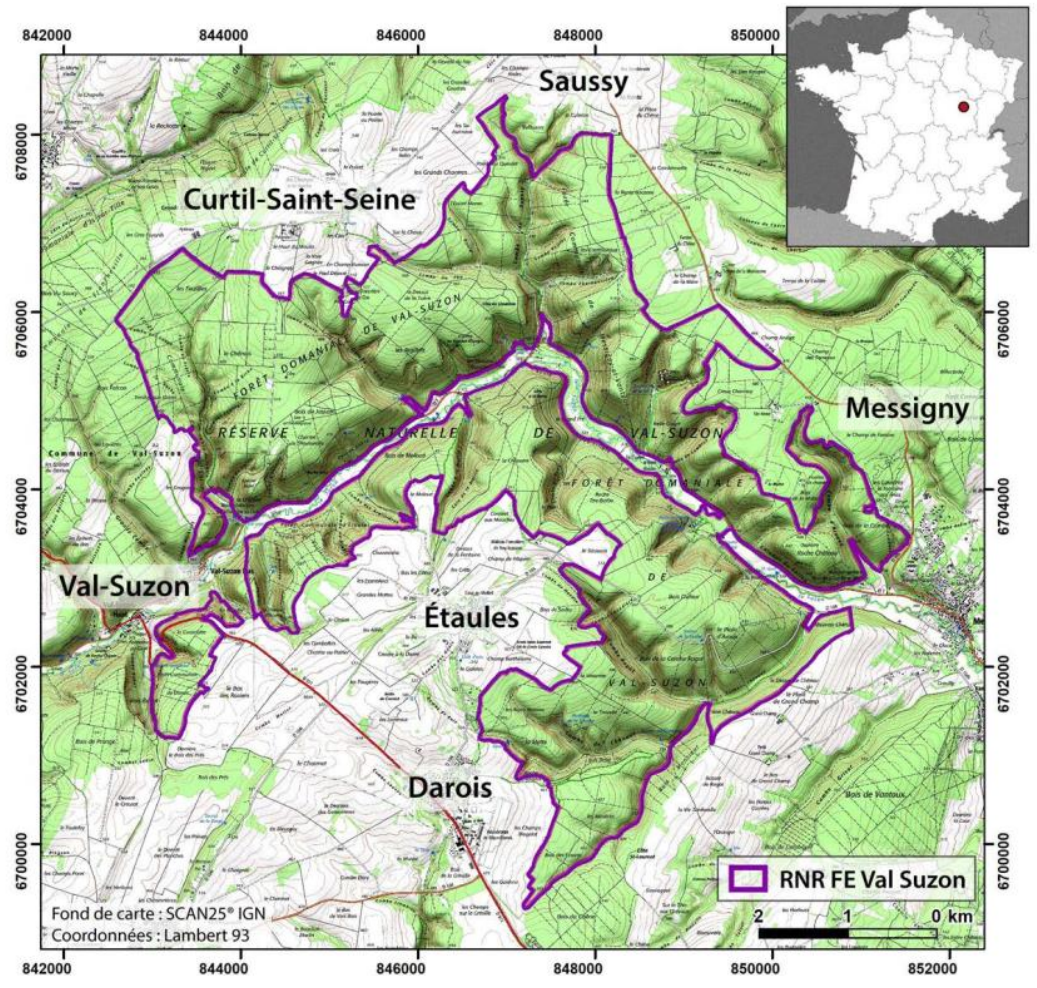

Figure 1. Localisation de la réserve naturelle régionale et Forêt d'Exception du Val Suzon (C R. Landois)

\section{Problématique \& sources}

À travers une approche pluridisciplinaire, ces recherches visent à identifier et étudier les dynamiques naturelles et sociales qui ont façonné la forêt du Val Suzon. Ce travail s'intéresse en effet à des problématiques relatives à l'environnement, au paysage, à l'histoire ou encore à l'archéologie. Ces réflexions scientifiques s'appliquent à des échelles temporelles et spatiales différentes. L'un des premiers objectifs de ces recherches concerne la caractérisation des ressources naturelles disponibles sur le territoire du Val Suzon, ainsi que leur gestion et leur exploitation au cours du temps. Il s'agit

\footnotetext{
${ }^{1}$ Plus d'informations à propos du label « Forêt d'Exception ${ }^{\circledR}$ » sur le site de l'Office Nationale des Forêts : http://www1.onf.fr/foretexception/@@index.html

${ }^{2}$ Notamment dans le cadre d'une thèse intitulée « Éco-dynamiques d'un espace forestier et des activités humaines : la forêt du Val Suzon sur le temps long ", réalisée par Rémi Landois à l'UMR 6298 Artehis, sous la direction de Jean-Pierre Garcia (université de Bourgogne Franche-Comté) et de Corinne Beck (université de Valenciennes).
} 
d'analyser les usages et les pratiques mis en place par les communautés humaines à différentes époques, et de documenter les traces que ces activités ont laissées. Ces activités ont potentiellement eu un impact sur l'environnement passé du Val Suzon - que ce soient les milieux forestiers, les sols, etc. et qui peut être encore perceptible aujourd'hui sous la forme d'héritages ou d'arrières-effets ${ }^{3}$. L'ensemble de ces données permettra par exemple de reconstituer l'évolution des paysages et de l'occupation humaine d'un territoire qui n'a pas toujours été forestier.

Par ses aspects diachronique et pluridisciplinaire, cette approche utilise un ensemble de sources variées. En premier lieu, se trouvent évidemment les résultats du levé LiDAR, réalisé en 2013 pour le compte de $\mathrm{l}^{\prime} \mathrm{ONF}^{4}$ (figure 2). Le modèle numérique de terrain produit à partir de ces données possède une résolution de $50 \mathrm{~cm}$. Plusieurs traitements ont été appliqués pour analyser l'ensemble des anomalies micro-topographiques et identifier les potentielles structures d'origine anthropique: ombrages, ombrages multidirectionnels, pente, ouverture topographique positive et négative (Openess) (Doneus, 2013), indice de visibilité du ciel (Sky View Factor) (Kokalj et al., 2011 ; Zaksek et al., 2011) et relief local moyen (Local Relief Model) (Hesse, 2010) ${ }^{5}$. Grâce à la combinaison des images ainsi obtenues et intégrées à un SIG, toutes les anomalies topographiques identifiées sont vectorisées. Un ensemble d'informations relatives à la forme, au relief, à la localisation, etc. sont renseignées dans une base de données, afin de compiler les éléments descriptifs avant l'étape d'interprétation. S'ensuit un travail de classification des structures archéologiques, et lorsque cela est possible, de datation et de typologie. Une fois les vestiges recensés, il est également nécessaire de vérifier leur nature in situ et, éventuellement, de récolter des informations supplémentaires grâce aux méthodes classiques en archéologie : prospections, sondages, etc.

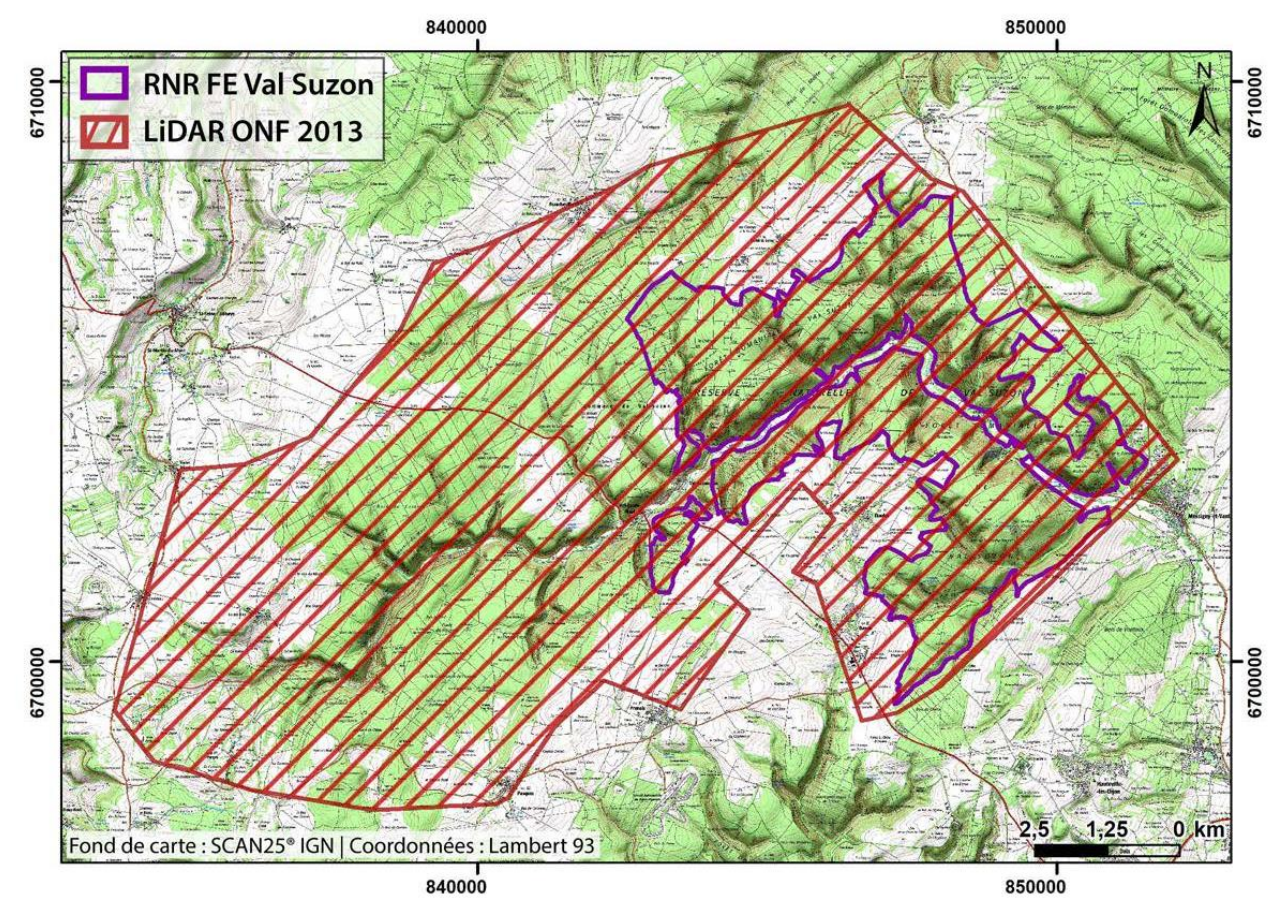

Figure 2. Emprise du levé LiDAR réalisé en 2013 sur une partie du bassin versant du Suzon (@ R. Landois)

\footnotetext{
${ }^{3}$ Des sujets qui sont notamment abordés en écologie historique (Decocq, 2018 ; Bergès \& Dupouey, 2017).

${ }^{4}$ Levé LiDAR réalisé par l'entreprise Sintégra, grâce au financement de la RNR, FEDER, sites classés et Forêt d’Exception ${ }^{\circledR}(\mathrm{ONF}, 2016$, p. 36).

${ }^{5}$ Ces traitements sont ceux habituellement utilisés en archéologie forestière à l'image de plusieurs autres forêts françaises étudiées suivant des protocoles tout à fait similaires, comme par exemple pour le Châtillonnais (Chevigny et al., 2015 et 2019). Les traitements ont été réalisés sur deux logiciels : Relief Visualization Toolbox conçue par I'Institut of Anthropological and Spatial Studies du centre de recherche de l'Académie des sciences et des arts de Slovénie (Kokalj \& Somrak, 2019 ; Zaksek et al., 2011) et le logiciel ENVI édité par la société ITT Visual Information Solutions.
} 
D'autres sources sont également exploitées pour interpréter et dater les structures repérées sur le LiDAR. C'est le cas des données archéologiques produites sur des sites déjà connus par le passé et qui ont fait l'objet de fouilles. Les archives textuelles modernes et médiévales peuvent également contenir des informations précieuses sur les vestiges présents dans le Val Suzon ${ }^{6}$. Ces archives sont riches de renseignements sur des activités qui n’ont pas nécessairement laissé de traces détectables par le LiDAR, comme l'exploitation forestière ou la chasse. Évidemment, les archives sont surtout indispensables pour comprendre le contexte historique du Val Suzon. Les documents qui sont étudiés dans le cadre de ces recherches sont pour la majeure partie conservés aux archives départementales de la Côte-d'Or, ainsi qu'aux archives municipales de Dijon et quelquefois par les mairies des différentes communes du Val Suzon. En plus des textes, il existe de nombreux plans anciens, qui concernent surtout les forêts et les bois. Les premiers plans datent du XVII ${ }^{\mathrm{e}}$ siècle et constituent des sources d'information remarquables pour reconstituer l'évolution de l'emprise forestière depuis la période moderne (Cousins, 2001 ; Dupouey et al., 2007 ; Dupouey et al., 2016; Vallauri et al., 2012). D'autre part, plusieurs sondages ont été réalisés dans la vallée et quelques combes du Val Suzon afin d'étudier les archives sédimentaires (Landois et al., 2020). Enfin, les recherches menées sur l'histoire Val Suzon s'appuient sur des données concernant l'environnement actuel de ce territoire forestier, c'est-à-dire des données pédologiques, écologiques ou phytosociologiques. Celles-ci sont généralement le fruit des travaux de l'ONF (carte des stations forestières, etc.) ou des autres scientifiques qui participent à l'amélioration des connaissances sur la réserve (ONF, 2016, p. 31).

\section{L'exemple du plateau de Messigny}

Quelques structures archéologiques recensées sur le plateau de Messigny seront présentées ici afin d'illustrer la problématique développée plus largement sur l'ensemble du territoire du Val Suzon. Le secteur qui a été sélectionné à titre d'exemple se situe sur la commune de Messigny, à $4 \mathrm{~km}$ au nord du village (figure 3). Ce secteur correspond à une zone de plateau calcaire qui surplombe à l'ouest la vallée du Suzon, et dont l'altitude ne dépasse pas les $525 \mathrm{~m}$. Il s'étend depuis la Combe Pavenole à l'est, à la Combe de Lambrun au sud, jusqu'à la Combe Charrière au nord et couvre ainsi une zone d'environ 200 hectares. Cette fenêtre d'étude est centrée sur les parcelles forestières dites du «Grand Continent » et de la «Mansenne », appartenant aux bois communaux de Messigny et comprises dans le périmètre de la RNR FE Val Suzon. Ces deux parcelles sont encadrées par des bois privés : des peuplements de feuillus au nord, tout à fait semblables aux bois communaux, et des plantations de résineux au sud.

\footnotetext{
${ }^{6}$ Par exemple, dans le Val Suzon, l'identification de garennes à lapins au lieu-dit des « Maisons Blanches » s'est faite notamment grâce aux archives textuelles et à un travail de confrontation des sources (Landois et al., 2019a).
} 


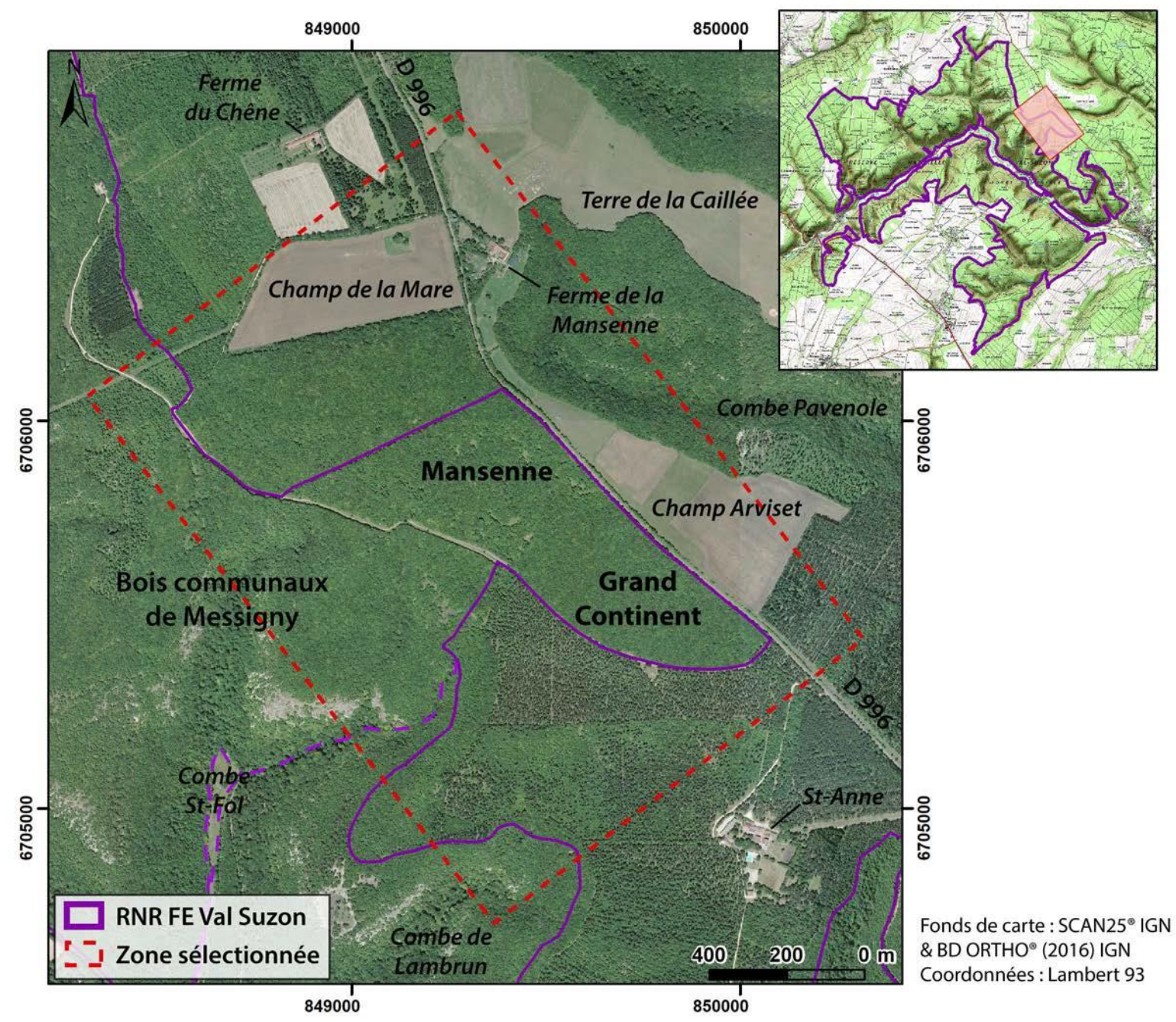

Figure 3. Présentation du secteur étudié sur le plateau de Messigny (commune de Messigny-et-Vantoux) (C) R. Landois)

\section{Historique de la forêt depuis le XVI siècle :}

Cet historique vise à reconstituer l'évolution de l'emprise forestière jusqu'à son état actuel, à partir de différents documents : des plans anciens et modernes, des photographies aériennes, des archives textuelles, etc. Ce travail s'effectue généralement de manière régressive, à partir des données récentes les plus complètes jusqu'aux premières représentations cartographiques du massif forestier datant de la fin du XVII ${ }^{\mathrm{e}}$ siècle. Il est parfois possible de remonter à des périodes plus anciennes grâce aux textes (arpentage des bois, mentions de limites et de confins, etc.), mais les sources s'avèrent souvent fragmentaires et les indications peu précises. Cette étude historique de la forêt permet à la fois d'identifier des tendances générales qui affectent l'ensemble du massif, et des dynamiques spécifiques à une commune ou à un bois. Ces informations sont parfois essentielles pour l'interprétation et la datation de structures archéologiques découvertes en contexte forestier, ou encore pour expliquer leur état de préservation. L'exemple le plus évident est celui des labours qui ont pu araser ou effacer entièrement les traces d'activités humaines antérieures. De la même manière, certaines plantations de résineux ont fait l'objet de travaux tout aussi destructeurs (réalisation de tranchées à l'aide de soussoleurs ou de charrues forestières par exemple).

En ce qui concerne le plateau de Messigny, le statut des bois et les types de peuplements rencontrés reflètent parfaitement les différentes trajectoires qu'ont suivies ces espaces au cours des siècles. En 1565, le chambrier de l'abbaye de Saint-Bénigne à Dijon, alors seigneur de Messigny, est à l'origine d'une importante métamorphose de l'occupation du sol sur le plateau. En quête de fonds pour des 
travaux de rénovation, celui-ci entreprend de faire défricher une partie des bois alors présents, sous prétexte que «la plus grand part sont petits bois aboudries, scitués et asscis sur roches et cottaults de montaynoi, ne pouvant prendre accroissance commodement pour la sterilite desdits lieux, remplis de mousses, ronces et buissons aubespins et autres bois empeschant laccroissance d'iceux de sorte que mal commodement et sans les laisser longuement sans coupper lon ny pouroit faire proffit en bois de taillis » (ADCO $1 \mathrm{H} \mathrm{814,} 3$ septembre 1566). Pour ce faire, en échange d'une redevance financière, il accorde à plusieurs bourgeois de la ville de Dijon le droit de s'installer sur les terres de Messigny, d'y établir des fermes, «a la charge de faire essarter [les bois] et mestre en nature de terre labourable »

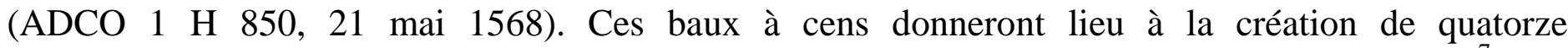
établissements agricoles, appelés «rentes», sur les hauteurs de Messigny, entre 1565 et $1568^{7}$. Les autres communes du Val Suzon n'ont visiblement pas connu d'épisodes de défrichements identiques à ceux de Messigny au XVI ${ }^{\mathrm{e}}$ siècle. Cette modification de l'environnement s'inscrit dans un contexte économique et politique particulier ${ }^{8}$. Dans un registre similaire, près de Val-Suzon, dans la partie amont dite du Val Courbe, se trouvent plusieurs habitats désertés dont la création serait liée à l'élevage de moutons pour le commerce de la laine entre le $X^{\prime} V^{\mathrm{e}}$ et le $\mathrm{XV}^{\mathrm{e}}$ siècle (Beck et al., 2018). Ce phénomène est pourtant cantonné au Val Courbe et n'affecte que marginalement le territoire du Val Suzon.

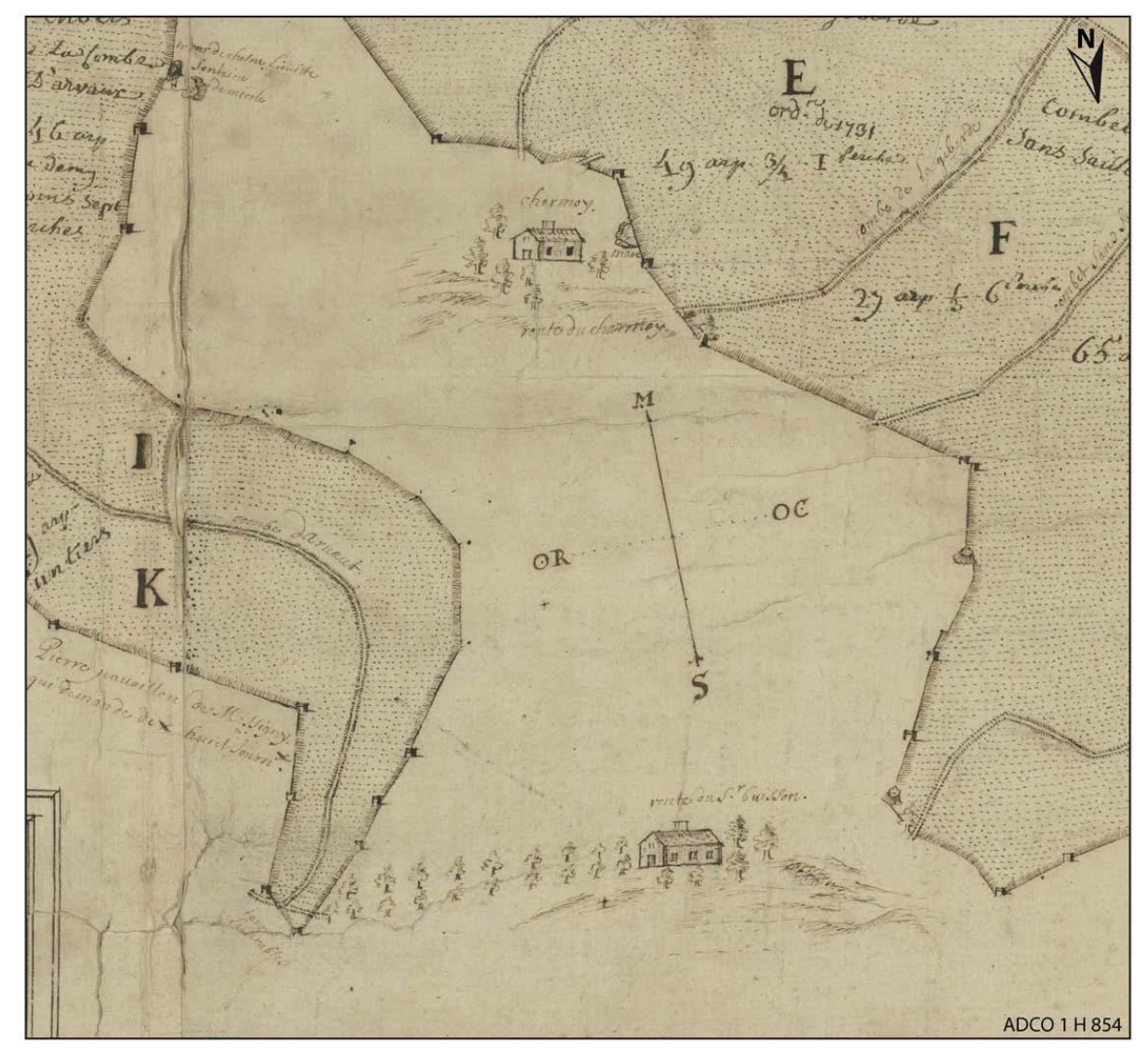

Figure 4. Extrait d'un plan de 1683 des bois de Messigny où sont dessinées deux « rentes », dites de «Charmoy » et du «sieur Buisson » (@ R. Landois)

\footnotetext{
${ }^{7}$ Pierre de Saint-Jacob évoque l'existence de 13 « métairies » sur le territoire de Messigny (de Saint-Jacob, 1995, p. 103) ; Jean Chiffre en dénombre 18 au total (Chiffre, 1982, p. 189).

${ }^{8}$ Pierre de Saint-Jacob rapproche cet épisode du phénomène plus général d'usurpation des communaux par « les abbayes, les seigneurs laïques, les parlementaires, les bourgeois, les marchands » (de Saint-Jacob, 1995, p. 103) ; Jean Chiffre y voit la volonté des abbayes de profiter de la « puissance foncière [de] la bourgeoisie marchande et la bourgeoisie parlementaire » et cite d'autres exemples de concessions similaires en Bourgogne (Chiffre, 1982, p. 189).
} 


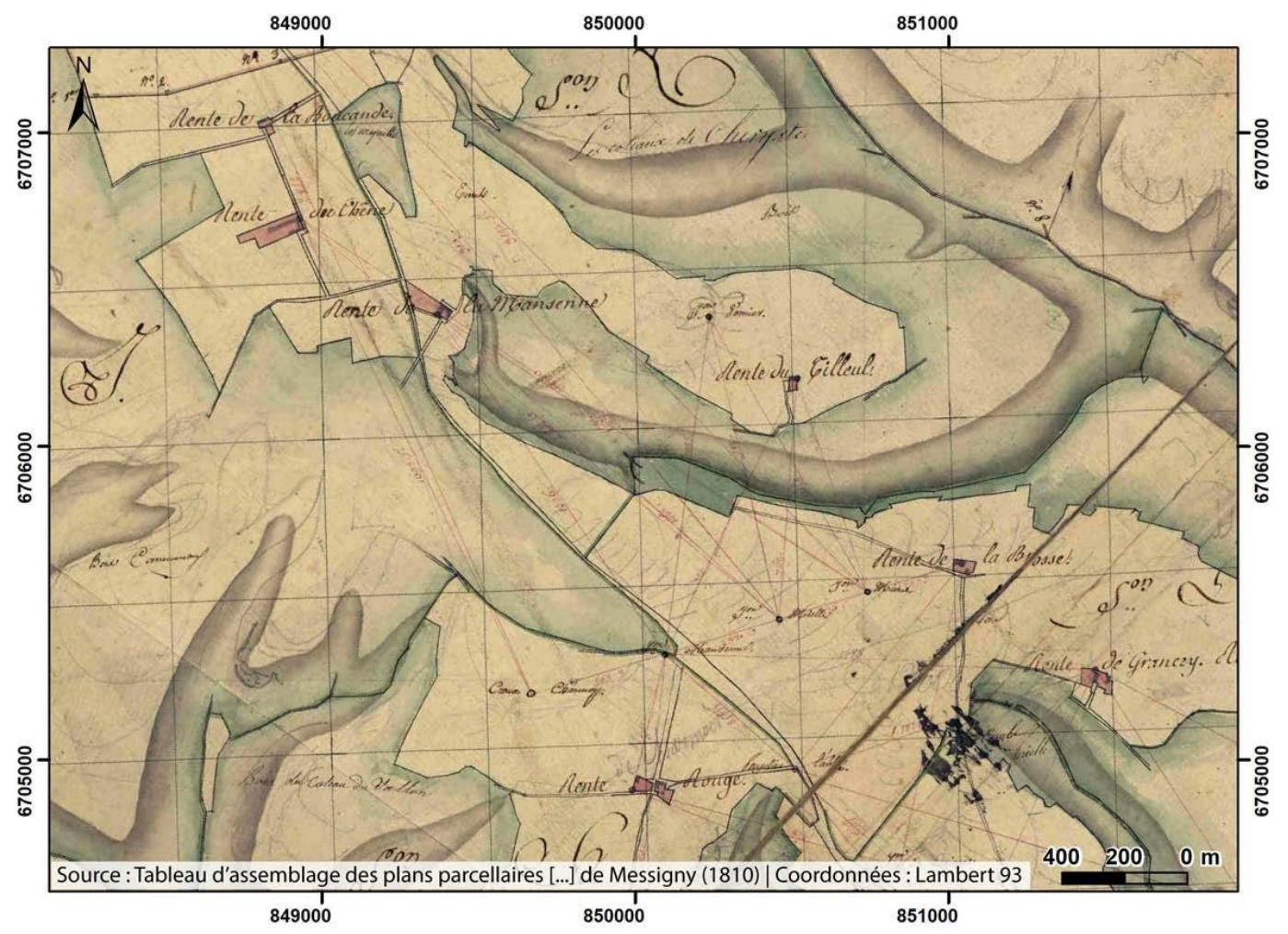

Figure 5. Extrait du cadastre napoléonien de Messigny; les différentes « rentes » y sont figurées en rose (C) R. Landois)

Grâce aux archives de l'abbaye de Saint-Bénigne9, il est possible de suivre finement l'évolution des fermes sur le plateau de Messigny, jusqu'à les retrouver sur les plans du XVII ${ }^{\mathrm{e}}$ siècle (figure 4). En 1810, il y a encore dix fermes visibles sur le cadastre napoléonien (figure 5). Si certaines « rentes » finissent par être abandonnées et ruinées, les terres attenantes continuent d'être exploitées ${ }^{10}$. À travers l'ensemble de ces sources, on constate finalement qu'au milieu du $\mathrm{XX}^{\mathrm{e}}$ siècle, l'espace occupé alors par les champs correspond presque entièrement aux terres défrichées quatre siècles plus tôt. Autrement dit, tout au long de cette période, l'emplacement de la lisière de la forêt n'a quasiment pas changé (figure 6). Ce n'est qu'à partir des années 1940 que le paysage sur le plateau de Messigny va à nouveau changer de manière notable. Les terres agricoles vont être progressivement remplacées par des plantations de résineux (figure 7). Durant les années 60, considéré comme une alternative à la production de bois de chauffage, l'enrésinement concerne aussi les forêts voisines. Il ne reste aujourd'hui que trois des anciennes « rentes » de Messigny et seule l'une d'entre elles possède encore une activité agricole (élevage de bovins, etc.). En fin de compte, on retrace l'évolution du paysage de Messigny, à travers la succession de différentes phases qui se rattachent plus généralement à des dynamiques ou à des modes de valorisation des plateaux calcaires. Si les bois communaux et la forêt domaniale de Messigny ne semblent pas affectés par ces événements, conservant leur état forestier, leur mode d'exploitation a parfaitement pu varier depuis le Moyen Âge (ONF, 2016, p. 23).

\footnotetext{
${ }^{9}$ Notamment grâce aux mémoires et aux cahiers de cens où sont reportées les redevances de chacune des fermes : ADCO 1 H 808,1 H 814, 1 H 850-851, 1 H 856.

${ }^{10}$ De petites concessions sont regroupées ensemble au cours du XVIII ${ }^{\mathrm{e}}$ siècle pour former de plus grandes exploitations (Chiffre, 1982, p. 190).
} 


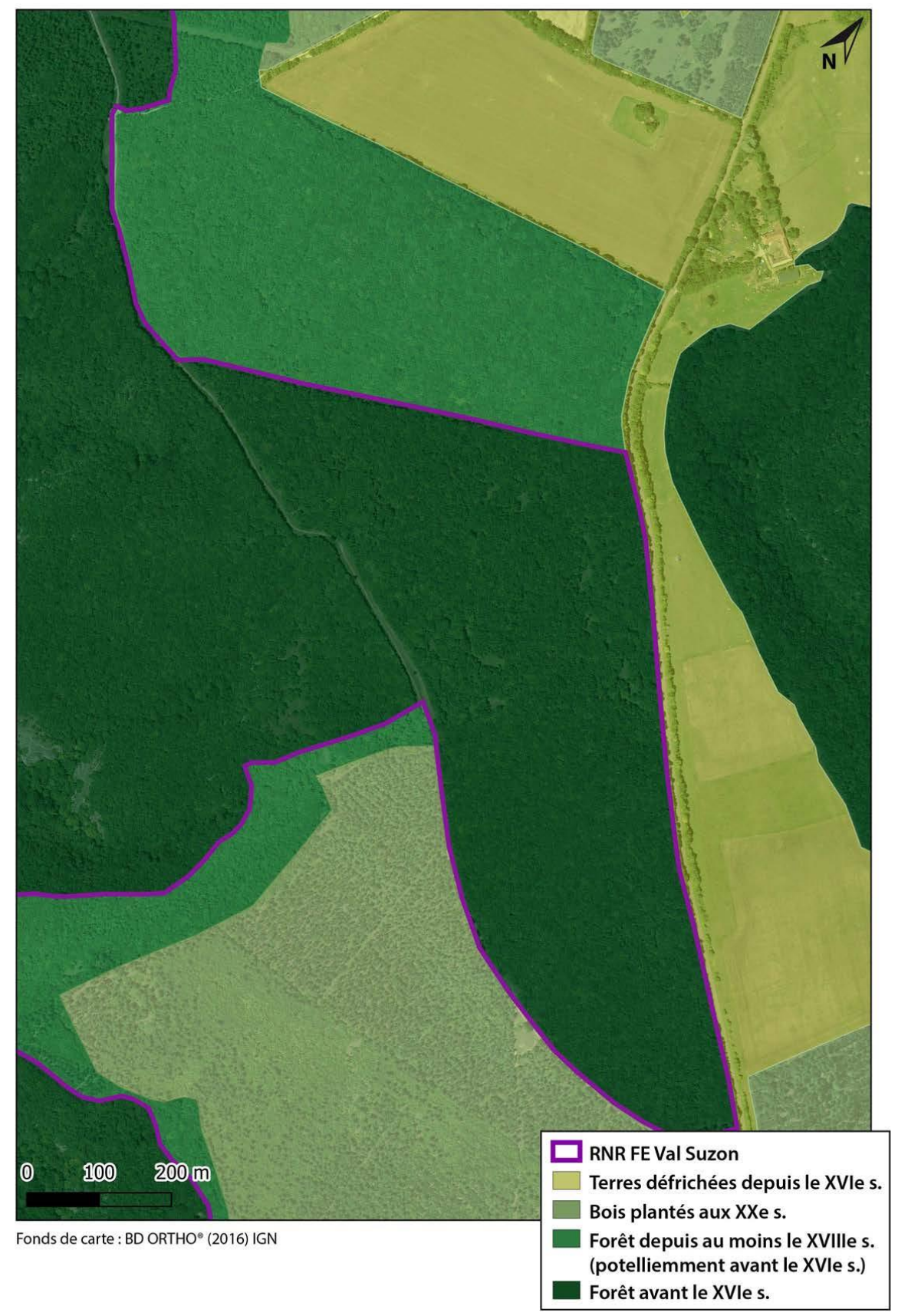

Figure 6. Carte de l'ancienneté des forêts sur le plateau de Messigny, réalisée à partir des plans anciens et des archives textuelles (C R. Landois) 


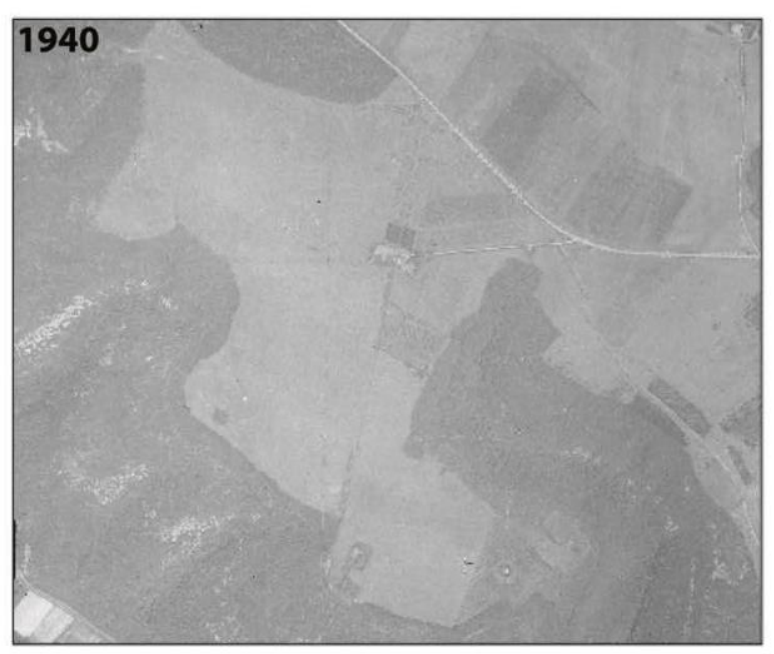

\section{3}
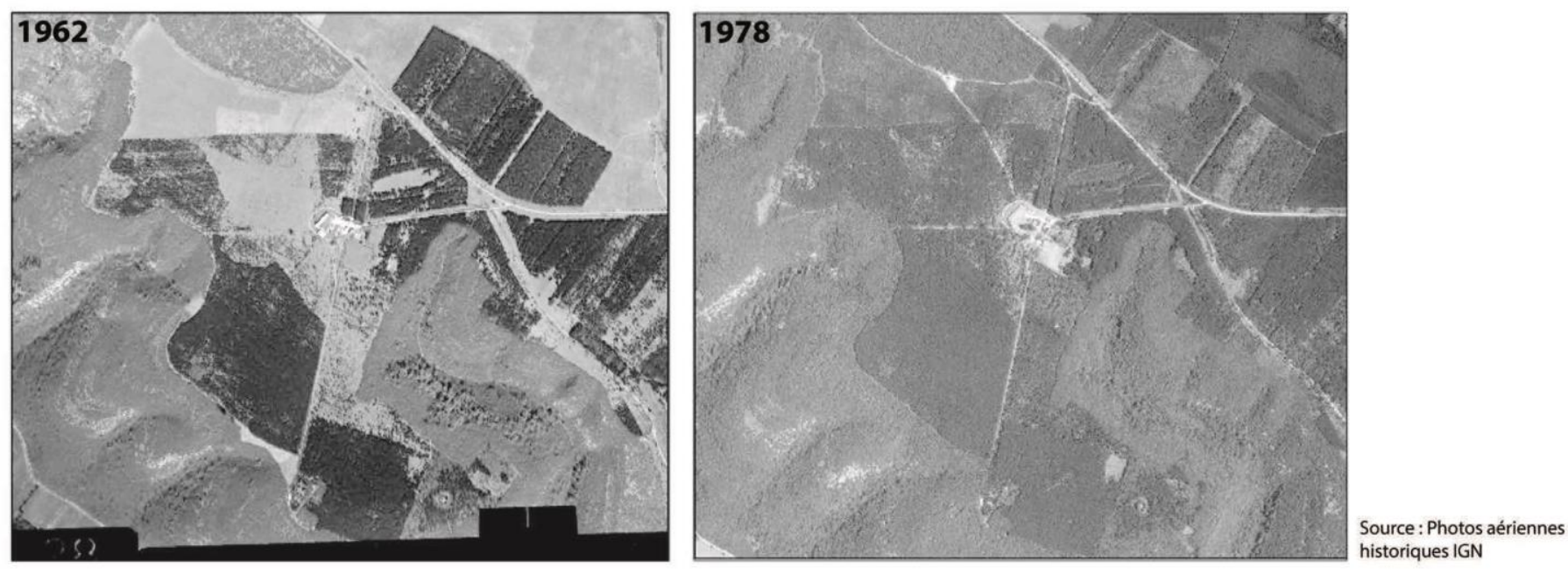

Figure 7. Photographies aériennes de la partie sud du plateau de Messigny en 1940, 1953, 1962 et 1978 (C) R. Landois) 
Présentation de quelques structures:

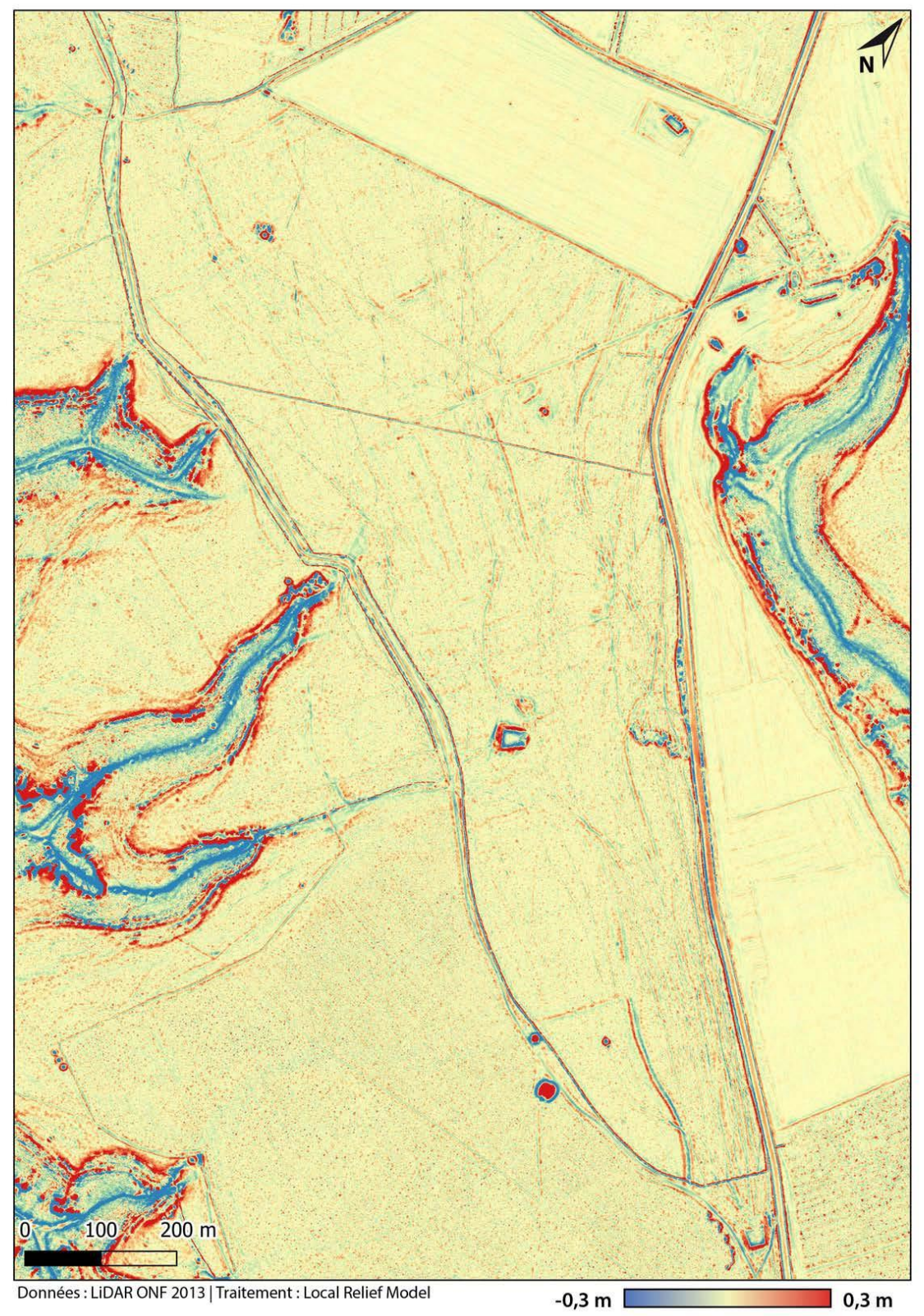

Figure 8. Extrait des données LiDAR sur le plateau de Messigny (@ R. Landois).

\section{Le tumulus de la Mansenne :}

Les premières découvertes archéologiques dans le Val Suzon sont bien évidemment antérieures à l'exploitation des données LiDAR. Parmi ces sites déjà connus depuis la fin du XIX $\mathrm{X}^{\mathrm{e}}$ siècle, se trouve le tumulus de la Mansenne, dans la parcelle de bois éponyme (figure 9). Il présente une circonférence d'environ $60 \mathrm{~m}$ et une hauteur maximale de 1,40 m. Fouillé par R. Brulard en 1909, il a révélé la présence d'une sépulture centrale contenant deux squelettes féminins et du mobilier (Brulard, 1909). On observe aujourd'hui encore les traces de cette fouille ancienne, sous la forme d'une tranchée creusée au centre de l'important monticule de pierres. Cette méthode d'exploration semble avoir été largement employée dans le Val Suzon, comme en témoignent les nombreux tertres perturbés semblables à des cratères ${ }^{11}$. Néanmoins, il n'est pas certain que toutes ces structures aient un jour

\footnotetext{
${ }^{11}$ Ces structures peuvent parfois être confondues sur les données LiDAR avec des fours à chaux (cf. chapitre « Charbonnières et fours à chaux »).
} 
possédé une fonction funéraire. La totalité des tertres qui ont été sondés par le passé ne sont pas mentionnés dans la littérature, au contraire du tumulus de la Mansenne et de celui de Jossam (commune de Val-Suzon), tous deux fouillés par R. Brulard, et qui ont livré des sépultures et du mobilier hallstattien (Provost, 2009, p. 526 et p. 230). Des fouilles infructueuses sont parfois évoquées dans les mémoires de la Commission des antiquités de la Côte-d'Or, sans qu'il ne soit donné plus de détails sur la localisation des structures, sinon qu'il s'agit généralement de tertres de petite taille (diamètres inférieurs à 10 m). Dès 1910, C. Drioton s'interroge déjà sur l'origine de ces tas de pierres : s'agit-il de «tombelles » dont les sépultures ont été arasées, ou bien de cabanes effondrées, ou encore de simples tas d'épierrement (Drioton, 1910, p. 233)? Ainsi, faute de fouilles systématiques, les regroupements de tertres sont volontiers considérés comme des nécropoles tumulaires. Par exemple, dans la moitié orientale du plateau de Messigny, sur les Coteaux de Chéry et les Terres de la Caillée, G. Grémaud et P. Lebel ont repéré en 1936 plus d'une dizaine de «tumulus en pierres sèches », interprétés comme tels, probablement par analogie avec le tumulus voisin de la Mansenne (CACO, 1939, p. 282). À l'heure où l'analyse des données LiDAR contribue à la découverte de nouvelles structures en pierres sous forêt, ces précédentes interprétations mériteraient d'être questionnées. On doit citer à ce sujet les travaux d'Y. Pautrat et A. Charmot sur plusieurs tertres dans le Châtillonnais. À la suite de sondages archéologiques, ils se sont finalement révélés être des tas d'épierrement (Pautrat \& Charmot, 2013).

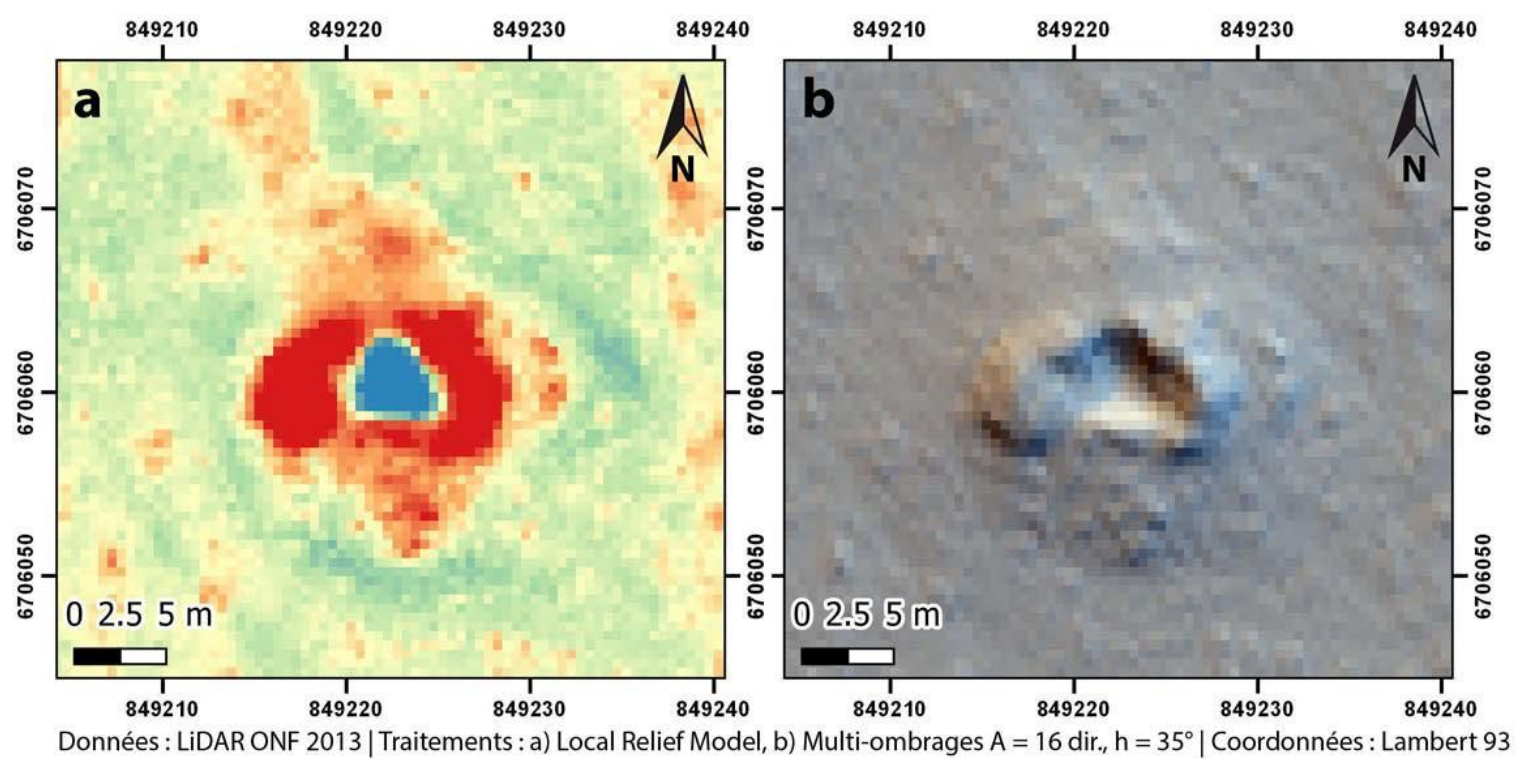

Figure 9. Extraits des données LiDAR pour le tumulus de la Mansenne (@ R. Landois)

Le tumulus de la Mansenne peut être rapproché d'autres sites archéologiques connus depuis longtemps dans le Val Suzon. Outre des tumulus, on trouve sur les plateaux calcaires plusieurs éperons barrés occupés durant le Néolithique et la Protohistoire : le Châtelet de la Fontaine au Chat sur la commune de Val-Suzon, le Châtelet d'Étaules sur la commune du même nom et la Roche-Château sur la commune de Messigny. Ces sites qui surplombent le Suzon ont notamment fait l'objet d'opérations archéologiques dès la fin du XIX ${ }^{\mathrm{e}}$ siècle, par C. Drioton (Drioton, 1910, p. 230) puis au cours des années 70 par J.-P. Nicolardot (Nicolardot, 2003). Ces éperons barrés correspondent à des prolongements du plateau, généralement cernés par des escarpements naturels et dont l'accès est barré par un système de remparts et de fossés. À l'instar des grands tumulus évoqués précédemment, ces fortifications sont aisément remarquables dans le paysage : à titre d'exemple, l'énorme amas de blocs du Châtelet d'Étaules mesure plus de $9 \mathrm{~m}$ de haut sur une surface de près de $2500 \mathrm{~m}^{2}$. La fonction de ces structures imposantes laisse peu de doute comme l'indiquent les toponymes présents dès le XVII ${ }^{\mathrm{e}}$ siècle sur les plans anciens. Il n'est guère surprenant que ces sites archéologiques aient été les premiers à attirer l'attention des savants du XIX ${ }^{\mathrm{e}}$ siècle de par leur taille. De plus, les fouilles menées par le passé sur les tertres suspectés d'abriter des sépultures, comme les stratégies employées (tranchées), 
traduisent les intérêts de l'époque. Ces derniers ont contribué à entretenir un biais de la recherche archéologique en forêt, en l'orientant d'abord vers des structures faciles à repérer et en mesure de livrer du mobilier. Mais ces découvertes archéologiques anciennes comme celles réalisées plus récemment grâce au LiDAR, pourraient également signaler un biais de préservation concernant les vestiges les plus anciens. En effet, les remparts et les tumulus constituent des reliefs en pierre importants, qui nécessiteraient des efforts conséquents pour être totalement démantelés au regard de l'espace qu'ils occupent. Idem en ce qui concerne leur dislocation et leur érosion naturelle. À l'inverse, d'autres structures qui leur seraient contemporaines, aux reliefs moins marqués, sont plus susceptibles d'avoir été complètement arasées ou enterrées, et par conséquent n'ont laissé aucune trace en surface. En l'état actuel, dans le Val Suzon, l'étude de l'organisation du territoire pour les périodes les plus anciennes s'appuie principalement sur l'utilisation de travaux antérieurs et se limite à l'identification de vestiges ponctuels (habitat et funéraire), sans qu'il soit possible de dessiner un schéma plus précis de l'occupation humaine passée.

\section{Parcellaires et champs bombés :}

À l'inverse des structures précédentes, certaines anomalies topographiques réparties sur de plus larges espaces permettent d'identifier d'anciennes phases d'occupation du sol, qui ont précédé l'état forestier actuel. Il s'agit d'ensembles de structures linéaires ou courbes, construites en pierre ou en terre, délimitant parfois des enclos fermés et qui constituent de vastes parcellaires aujourd'hui préservés sous forêt. Le vocabulaire pour décrire ces structures est relativement riche et souligne la diversité des formes que présentent ces objets : murées, talus, terrassements, épaulements, etc. À travers ces termes, transparaissent également les différents processus d'édification de ces limites. S'ajoutent à ces lignes des structures plus ponctuelles comme les tas d'épierrement, parfois répartis à l'intérieur d'un enclos ou bien alignés dans le prolongement d'une murée. De manière générale, on associe ces parcellaires à des pratiques agro-pastorales, suivant un découpage et une organisation du territoire sur des périodes plus ou moins longues ${ }^{12}$. Dès lors, la datation de ces parcellaires peut s'avérer assez complexe, la forme des parcelles n'étant pas un argument suffisant pour attribuer cette occupation à une période donnée ${ }^{13}$. Néanmoins, certains sites domestiques, cultuels ou funéraires s'insèrent de manière cohérente à l'intérieur de ces parcellaires, ce qui indiquerait une probable contemporanéité. Ces sites sont alors généralement plus propices à apporter des informations sur l'âge et les modalités d'occupation de ce territoire. L'un des premiers exemples que l'on peut citer de parcellaires mis au jour à partir de l'analyse des données LiDAR est sans doute celui de la forêt de Haye près de Nancy (Georges-Leroy et al., 2012 ; Georges-Leroy et al., 2014). Dans le même registre, l'exemple du Châtillonnais, où les recherches avaient débuté en 1995 par de simples prospections pédestres et des relevés GPS, illustre parfaitement l'apport de la technologie LiDAR pour cartographier ces grands ensembles (Goguey et al., 2010). À l'image d'autres forêts françaises, le Val Suzon a également livré son lot de structures agraires. Ainsi, sur le plateau de Jossam, se trouve un vaste parcellaire sur près de 400 hectares exploité au moins jusqu'à l'Antiquité (Landois et al., 2020). Sur le plateau voisin dit de Goa, un village médiéval et son finage ont pu être identifiés, notamment grâce à des documents d'archives textuelles du XII ${ }^{\mathrm{e}}$ siècle et à la découverte de mobilier céramique lors de prospections (Landois et al., 2019b). On rencontre aussi sur les plateaux quelques indices de l'extension maximale de l'aire cultivée autour des villages de Darois et d'Étaules, sous la forme de terrasses et de rideaux de culture. Ces vestiges, qui n'ont pu être datés pour l'instant, matérialisent ainsi l'évolution de l'emprise forestière dans le passé.

\footnotetext{
${ }^{12}$ On peut d'ailleurs s'interroger sur le temps nécessaire à la mise en place des parcellaires et notamment à l'érection de certaines limites. À ce sujet, on peut évoquer le travail mené sur des murées en Alsace à partir d'estimations du volume des structures
}

(Schwartz et al., 2012, p. 322).

${ }^{13}$ Hormis les cas particuliers, comme celui des centuriations (Favory, 2012, p. 115). 
En ce qui concerne le territoire de Messigny, l'occupation moderne des plateaux a largement contraint la préservation de grands ensembles parcellaires. Si quelques murées et tertres d'épierrement sont bien présents dans la moitié nord de la commune, la partie la plus méridionale des plateaux calcaires en est quasi totalement dépourvue. En effet, cette zone est surtout occupée par des plantations de résineux, présentées plus tôt, et des versants assez abrupts, peu propices à l'implantation de structures parcellaires. Néanmoins, aux lieux-dits du Grand Continent et de la Mansenne, la forêt a préservé des traces uniques à l'échelle du Val Suzon (figures 8 \& 10). Celles-ci dessinent des ensembles de lignes parallèles d'environ $10 \mathrm{~m}$ de large en moyenne, dont le relief ne dépasse pas $30 \mathrm{~cm}$ de hauteur, semblables à de légères ondulations topographiques. Ces formes, que l'on peut regrouper en fonction de leur orientation évoquent des champs bombés, comme ceux préservés par exemple sous les forêts du Pays de Bade (Sittler \& Hauger, 2007) ou sous la forêt de Haye (sous le nom de «billons»; Georges-Leroy, 2015, p. 12). Il pourrait également s'agir de rideaux de culture, mais étant donnée la faible pente de cette partie du plateau de Messigny, il est difficile de l'affirmer. D'après R. Sitller, ces traces sont celles de labours, exécutées à l'aide de charrues à versoir fixe (Sittler, 2015, p. 5). On aurait donc ici l'empreinte de pratiques agricoles vieilles de plus de 500 ans, puisque l'on sait qu'au $\mathrm{XV}^{\mathrm{e}}$ siècle ces plateaux étaient recouverts de bois et de friches, d'après les archives textuelles. D'ailleurs, c'est certainement le retour assez rapide de la végétation sur ces terres après leur abandon, qui a permis de préserver ces micro-reliefs. Au contraire, on devine difficilement le prolongement de ces champs bombés plus au nord, dans l'actuel Champ de la Mare: un espace défriché et mis en culture depuis le milieu du $\mathrm{XVI}^{\mathrm{e}}$ siècle, après la création de la ferme de la Mansenne. À cet endroit, on distingue d'autres ondulations, orientées $170^{\circ}$ nord, que l'on associe à l'exploitation moderne du Champ de la Mare (figure 10). Enfin, il est important de noter que la topographie générale de ce secteur - le sommet d'un plateau calcaire relativement plat et régulier - a favorisé la conservation de ces traces, en limitant les phénomènes d'érosion et de colluvionnement. Cette caractéristique topographique bénéficie aussi aux traitements d'imagerie menés sur les données LiDAR, en facilitant l'identification de reliefs de très faible amplitude et de grande longueur d'onde.

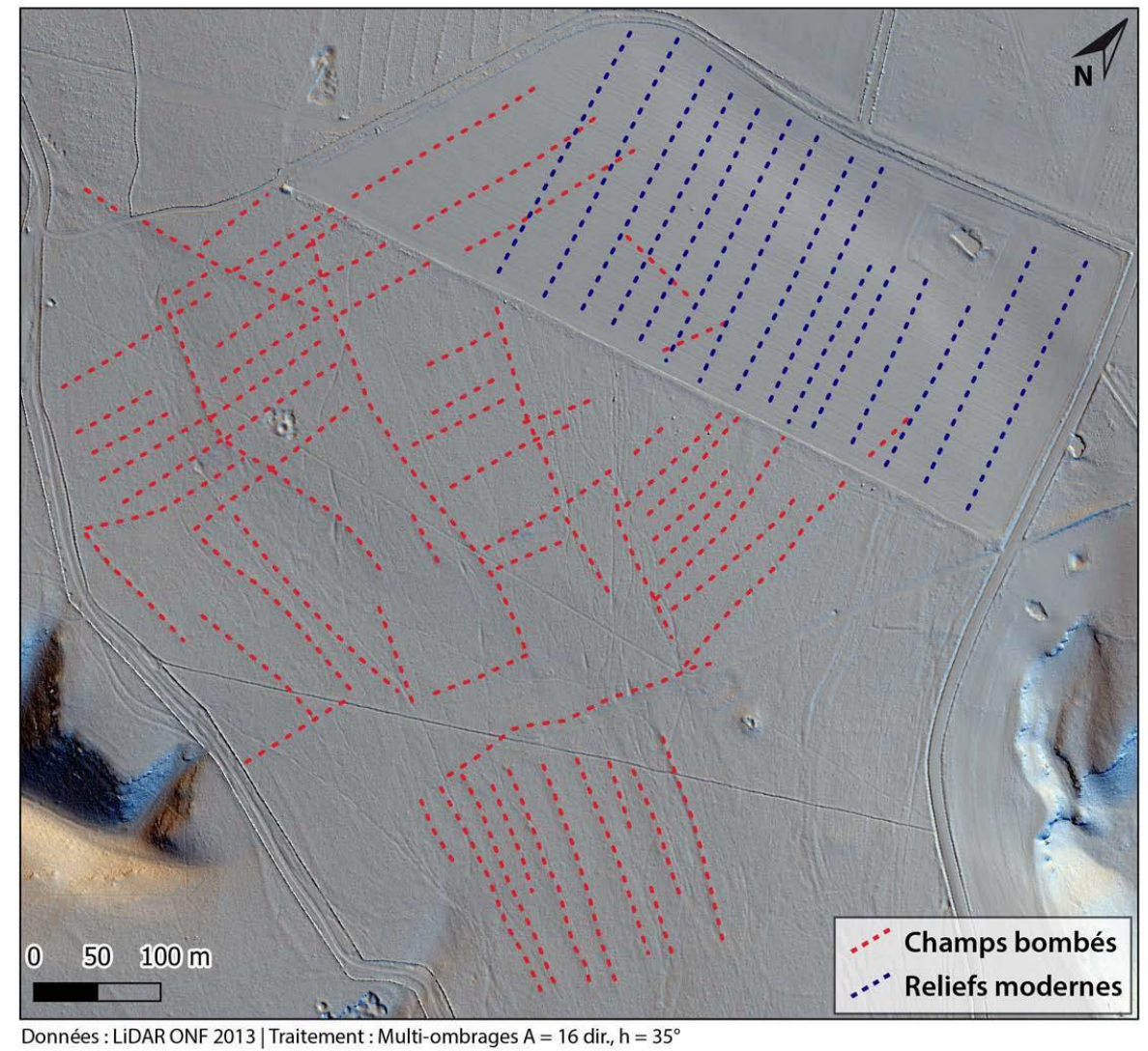

Figure 10. Traces de labours relevées d'après les données LiDAR (@ R. Landois) 


\section{Traces de passage d'animaux et réservoirs :}

Dans les parcelles de bois appelées le Grand Continent et la Mansenne, où se trouvent les champs bombés décrits ci-dessus, d'autres traces rarement décrites ont été repérées grâce au LiDAR alors qu'il est pourtant difficile de les percevoir toutes sur le terrain. Il s'agit de très nombreuses dépressions linéaires, assez étroites (moins de $2 \mathrm{~m}$ ) et peu profondes, qui s'étirent tout au long de ces bois (figures $8 \& 11$ ). Leur signature topographique se rapproche de celle des sentiers, les structures étant ici bien plus nombreuses et entrelacées. Leur trajectoire n'est pas totalement aléatoire : ces traces de passage dessinent des sortes d'itinéraires ou de parcours (figure 12). En premier lieu, toutes les traces partent d'un même point de départ situé à l'extrémité sud-est du bois de la Mansenne, se prolongent vers le nord-ouest puis se répartissent ensuite en trois fuseaux. Le premier, assez dense, poursuit son cheminement en obliquant légèrement vers le nord. Le deuxième groupe continue vers le nord-ouest dans la suite du segment initial, jusqu'à atteindre la lisière du bois du Grand Continent. Les traces du troisième et dernier groupe, moins nombreuses et plus espacées, se dirigent vers l'ouest, vers l'extrémité de la Combe Charrière, où elles finissent par se dissiper.

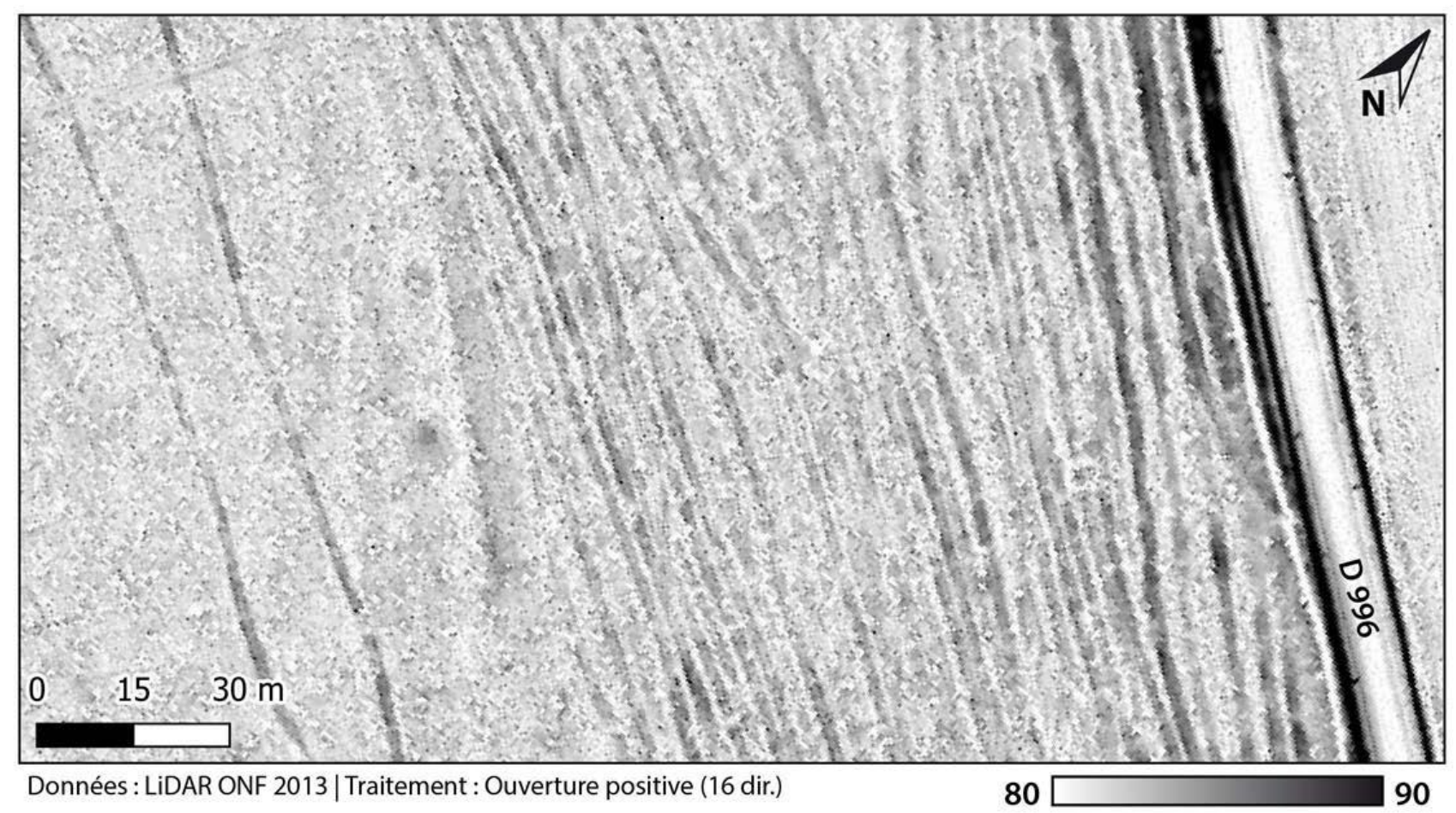

Figure 11. Extrait des données LiDAR sur les traces de passage d'animaux le long de la D 996 (@ R. Landois) 


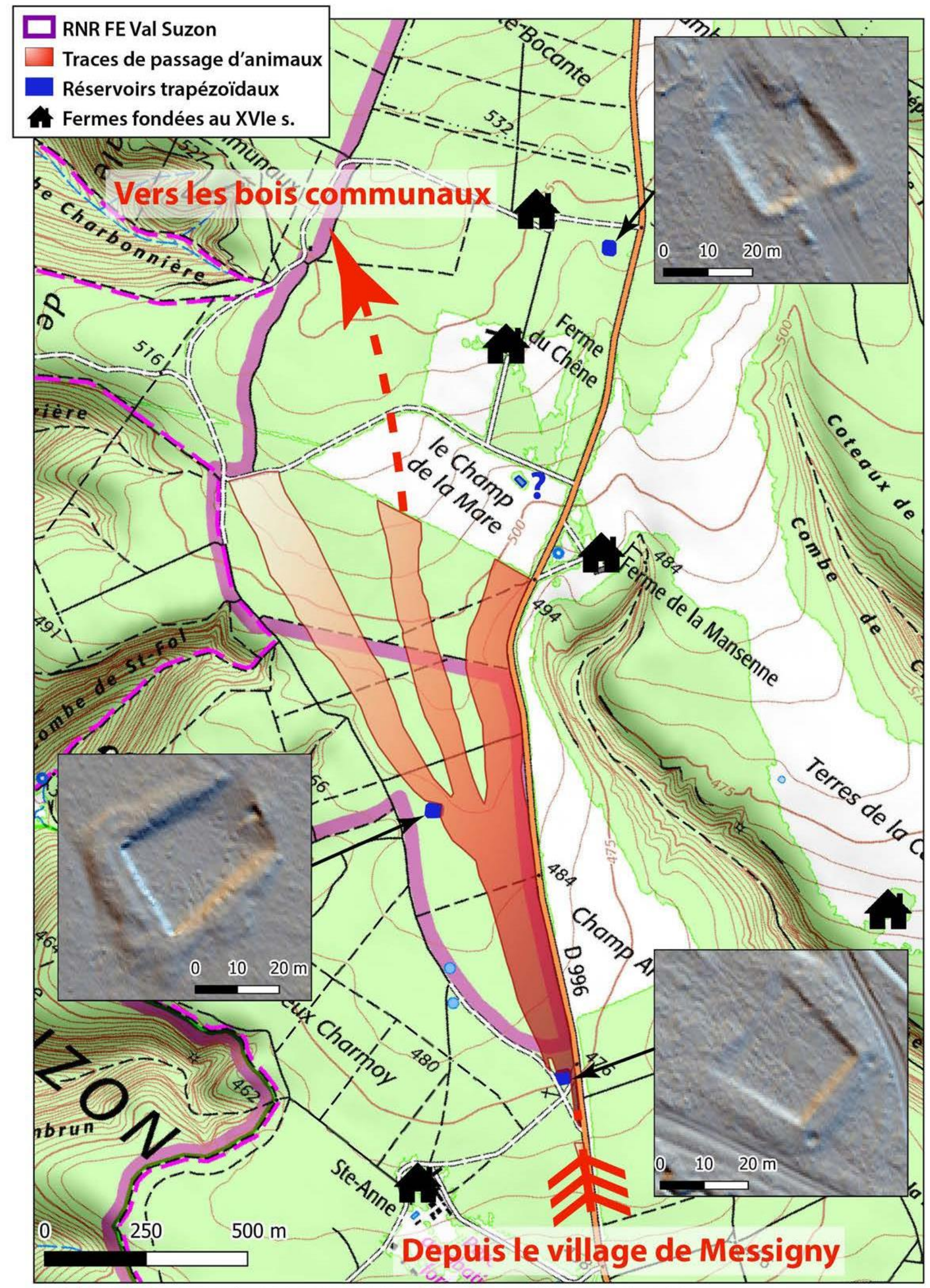

Fond de carte : SCAN25 $5^{\oplus}$ IGN | Données : LiDAR ONF 2013 | Traitement : Multi-ombrages (16 dir.)

Figure 12. Schéma de l'itinéraire potentiel suivi par les animaux sur le plateau de Messigny (@ R. Landois)

Ces traces convergent vers deux grandes structures trapézoïdales (figures 8 \& 12) visibles sur les données LiDAR et qui correspondent à des mares artificielles. Les processus karstiques limitent les réserves en eau des plateaux, ce qui a poussé leurs occupants à y installer des réservoirs pour recueillir l'eau des précipitations. Il s'agit en l'occurrence de structures creusées dans le substrat, dont le fond a 
pu être tapissé d'argile pour assurer une certaine étanchéité, et qui sont dotées d'un plan incliné pour en assurer l'accès au bétail. L'un de ces réservoirs est également pourvu d'une source de trop-plein maçonnée et protégée par une voûte en pierre (figure 13). Ces mares sont assez courantes au cœur des villages de la région, mais l'on en trouve ici au moins trois du même type installées sur le plateau de Messigny (figure 12). La plus septentrionale est dans un mauvais état de conservation : traversée par un chemin d'exploitation forestière, elle a été en partie comblée de gravats. Ces structures, dont l'une d'elles a probablement donné son nom au Champ de la Mare, ponctuent ainsi un itinéraire qui partirait du village de Messigny au sud, en direction du nord, vers les bois communaux ou vers le village de Saussy. Des traces de ce cheminement auraient été préservées à l'emplacement des bois du Grand Continent et de la Mansenne, mais totalement effacées ailleurs. À ce propos, on remarque que ces micro-reliefs ne sont pas visibles dans les champs et les bois privés qui bordent la forêt domaniale : ces traces sont par conséquent plus anciennes. À l'inverse, ces traces sont postérieures aux champs bombés présentés précédemment, puisqu'elles les recoupent en plusieurs endroits. Finalement, on obtient une datation relative des différentes structures, tout en sachant qu'elles témoignent d'activités antérieures au défrichement partiel du plateau de Messigny à partir du milieu du XVI ${ }^{\mathrm{e}}$ siècle.

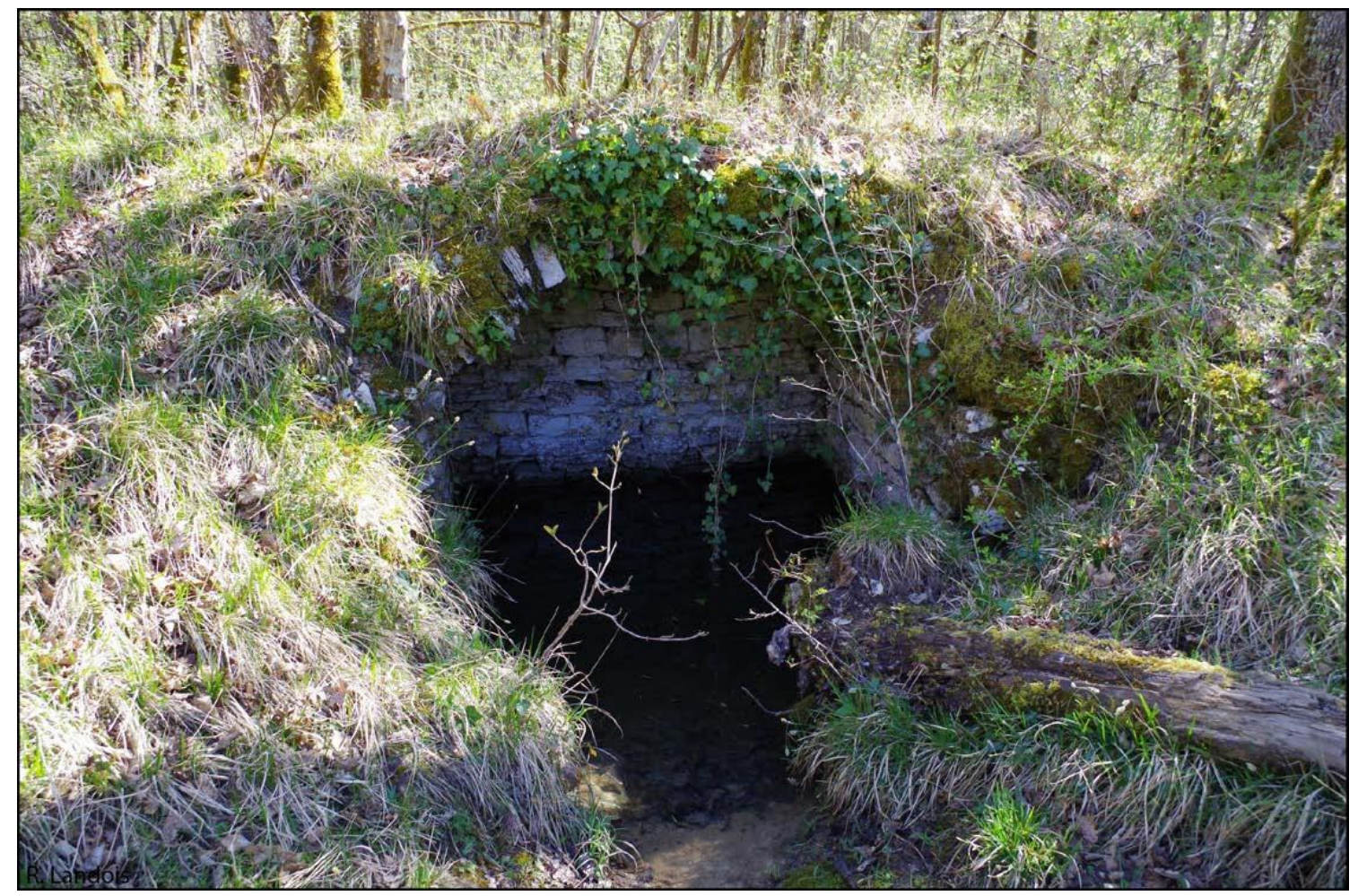

Figure 13. Photographie du puits installé dans l'un des réservoirs sur le plateau de Messigny (@ R. Landois)

À partir de l'ensemble de ces observations, on peut avancer plusieurs hypothèses quant à l'origine de ces traces. D'après leur nombre et leur densité, il pourrait s'agir du cheminement de bétails : le passage répété d'animaux qui se déplacent en groupe et qui se suivent aurait marqué durablement le sol. On pourrait par ailleurs objecter que ces traces ont pu être laissées par des animaux sauvages, puisqu'il leur arrive également d'emprunter préférentiellement certains itinéraires. Cependant, si l'on retrouve dans le Val Suzon des traces similaires près de points d'eau, celles-ci s'étendent sur de faibles longueurs et sont nettement moins nombreuses. Une autre hypothèse serait celle du passage de charrettes et autres véhicules, utilisés notamment pour aller puiser de l'eau dans les réservoirs et l'apporter aux fermes sur le plateau. Le tracé des chemins anciens habituellement empruntés par ces véhicules est généralement beaucoup plus régulier, ce qui ne correspond pas à l'importante divagation des traces visibles ici. La réalisation d'une coupe stratigraphique permettrait éventuellement de vérifier la présence d'ornières. D'autre part, aucune découverte dans les archives n'a permis pour l'instant de préciser les raisons de ces multiples cheminements d'animaux. Comme énoncé précédemment, l'itinéraire pourrait être celui emprunté par le bétail des habitants de Messigny, qui rejoint les bois 
communaux pour le pâturage en forêt ou les pelouses du plateau. Cette pratique figure en tout cas parmi les droits conférés aux habitants dans le terrier de Messigny établi en 1676 (ADCO 1 H 814). Cette coutume est peut-être même plus ancienne, puisque les habitants disposaient déjà de bois communaux au début du $\mathrm{XV}^{\mathrm{e}}$ siècle. On peut également imaginer que cet itinéraire ait été emprunté dans le cadre de la transhumance de cheptels depuis la plaine de la Saône vers la Montagne dijonnaise (de Saint-Jacob, 1995). La découverte de ces traces incroyablement conservées par la forêt soulève en fin de compte de nombreuses questions : quels sont les animaux impliqués, est-ce que leur parcours s'effectuait en forêt ou dans un milieu semi-ouvert, à quelle vitesse s'est réinstallée la forêt sur ces espaces, etc. Seule une étude pluridisciplinaire pourrait potentiellement apporter des éléments de réponse supplémentaires ${ }^{14}$.

\section{Charbonnières et fours à chaux :}

Toutes les structures décrites jusqu'ici correspondent à différents schémas d'occupation du territoire de Messigny, qui se sont succédé jusqu'à l'époque moderne. La structuration de cet espace est ainsi déterminée par la répartition des activités qui y prennent place et donc par l'exploitation de ses ressources. On trouve également dans les forêts du Val Suzon, d'autres structures, plus ponctuelles mais très nombreuses, qui sont notamment liées à l'utilisation des ressources sylvicoles. Certaines sont alors contemporaines de l'environnement forestier tel qu'on le connaît depuis le XVI siècle d'après les documents d'archives. C'est le cas des charbonnières ou plateformes de charbonnage, assurément les plus nombreuses dans le Val Suzon et dont l'origine remonte même au $\mathrm{XV}^{\mathrm{e}}$ siècle d'après certains indices toponymiques. Ces vestiges témoignent de la production de charbon de bois par cuisson «en meule », c'est-à-dire à partir d'un tas de bois recouvert de terre, de branchages et de feuilles, qu'on laisse carboniser ainsi pendant plusieurs jours. Une fois la cuisson terminée, le charbon est récolté et il ne subsiste plus à l'emplacement de la meule qu'une légère dépression circulaire parfois entourée d'un anneau périphérique étroit. Lorsque la topographie n'est pas suffisamment régulière ou plane, il a parfois été nécessaire d'aménager la place où devait être montée la meule. On trouve ainsi sur les versants ou en fond de vallée de véritables plateformes en terre ou en pierre ${ }^{15}$. Outre ces reliefs, l'identification de ces charbonnières sur le terrain est grandement facilitée par la couleur noire du sol et du fait de la présence de nombreux fragments de charbons. Ces structures représentent un sujet d'étude très riche et instructif sur l'histoire des forêts, il existe ainsi une littérature abondante sur ces objets (Paradis-Grenouillet et al., 2018). Ainsi, différents travaux sur les charbonnières du Val Suzon sont en cours ${ }^{16}$ et s'intéressent notamment à l'ampleur de cette activité sur ce territoire et à son potentiel impact sur l'environnement (Landois et al., 2017).

D'autres travaux sont menés autour de questions similaires concernant la production de chaux dans le Val Suzon. On rencontre notamment sur le plateau de Messigny différents exemples de fours à chaux, semblables à des cratères, quelquefois accompagnés de carrières (figures 8 \& 14). Ces fours sont constitués d'un monticule avec une dépression centrale à l'intérieur de laquelle se trouvent généralement des blocs de chaux et de calcaires calcinés. Comme pour les charbonnières, les fours à chaux sont suffisamment nombreux pour que l'on puisse étudier leur répartition et essayer d'établir une typologie. Par ailleurs, aucun exemple de four à chaux installé en dehors de la forêt n'a pour l'instant été découvert dans le Val Suzon. La proximité du bois, utilisé comme combustible pour la cuisson du calcaire, est certainement un critère majeur. L’implantation de ces structures est guidée par

\footnotetext{
${ }^{14}$ À travers la poursuite des recherches dans les archives communales, la réalisation de prélèvements et d'une étude microstratigraphique, des analyses de la matière organique, etc.

${ }^{15}$ Outre les autres modes de production de charbon de bois connus par ailleurs (en fosse par exemple), une typologie des traces laissées par les charbonnières en meule a notamment été définie pour les forêts de Charente (Rassat et al., 2018).
}

${ }^{16}$ Sondage archéologique, analyse anthracologique, analyse spatiale, estimation du volume de bois consommé, etc. 
la présence d'un substrat calcaire, dont l'exploitation en carrière paraît moins contraignante en forêt, épargnant ainsi les terres cultivées. D'ailleurs, plusieurs mentions dans les archives ainsi que des datations radiocarbone permettent d'affirmer que certains fours ont bien été établis en forêt. Il reste encore à déterminer à qui était destinée précisément cette production de chaux et s'il est possible de reconstituer une chronologie de cette activité pour le Val Suzon.

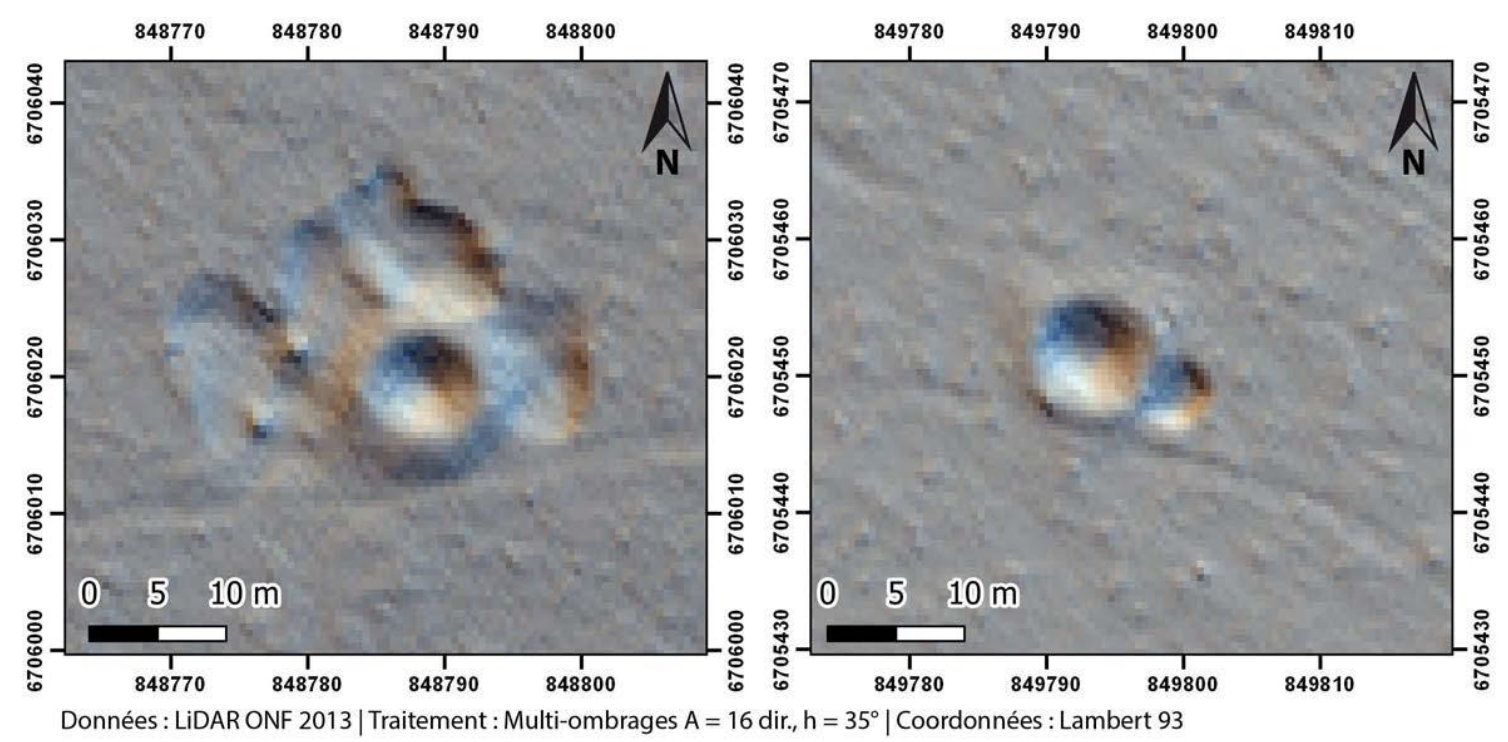

Figure 14. Deux exemples de fours à chaux découverts sur le plateau de Messigny (@ R. Landois)

\section{Tranchées de la guerre de 1870 :}

Pour terminer avec les exemples de structures ponctuelles, il y a sur les plateaux de Messigny et de Val-Suzon des tranchées datant de la guerre de 1870 (figures 8 \& 15). En effet, quelques escarmouches ont eu lieu autour de Dijon entre les troupes allemandes, françaises ou encore avec les francs-tireurs menés par Garibaldi. Par exemple, le 21 janvier 1871, lors de la troisième bataille pour la ville de Dijon, plusieurs combats sont rapportés dans le Val Suzon et aux alentours (Ledeuil d'Enquin, 1893, p. 35). Le Val Suzon, avec sa vallée étroite, ses multiples combes et ses versants boisés, constituait un terrain propice aux embuscades, au point d'être considéré «comme les Thermopyles ${ }^{17}$ de la Bourgogne» (Cremer \& Poullet, 1873, p. 122). Voilà sans doute pourquoi des tranchées ont été creusées à la lisière et à l'intérieur des bois. Celles-ci présentent d'ailleurs des formes très proches des tranchées de la Première Guerre mondiale visibles sur les données LiDAR de la forêt domaniale de Verdun (de Matos Machado et al., 2016). Avec le tumulus de la Mansenne, ces structures illustrent à la fois le potentiel des forêts comme milieu de préservation de vestiges archéologiques d'époques différentes, et celui du LiDAR comme technologie pour leur détection. De plus, les champs bombés et les traces de passage d'animaux retrouvés sur le plateau de Messigny sont le fait de l'accumulation et de la répétition d'une activité humaine, ce qui sous-entend une durée relativement longue. À l'inverse, les tranchées de la guerre de 1870 ont un caractère événementiel et tiennent même de l'éphémère. Bien que ces traces aient tout de même plus d'un siècle, elles sont toujours parfaitement identifiables dans le paysage. Encore une fois, le retour de la forêt a sans nul doute joué un rôle prépondérant dans leur préservation.

\footnotetext{
${ }^{17}$ En référence à la célèbre bataille des Thermopyles, ayant eu lieu en Grèce en 480 av. n. è., dans un défilé au pied des montagnes du Pinde.
} 


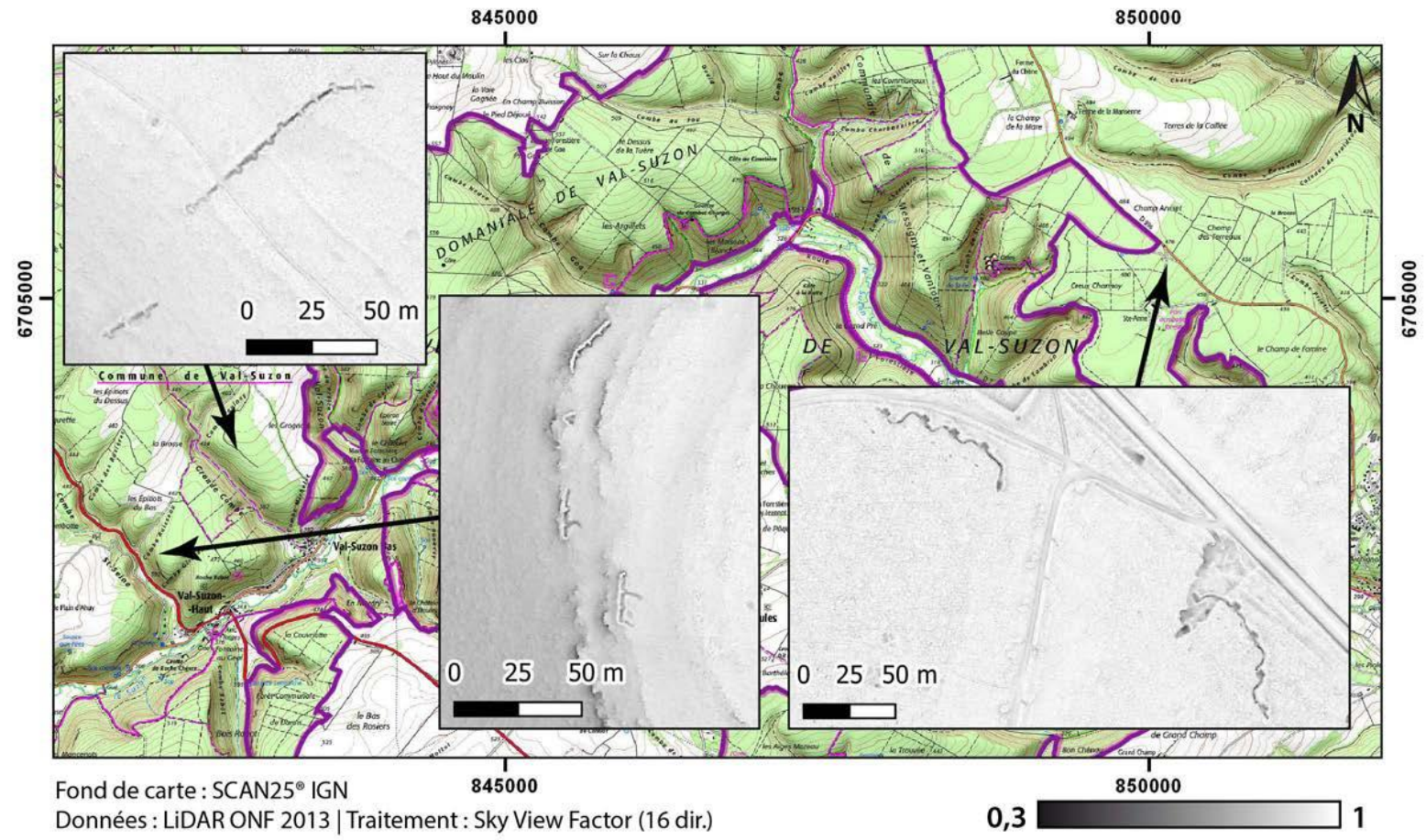

Figure 15. Extraits des données LiDAR sur les tranchées de la guerre de 1870 retrouvées dans le Val Suzon (C R. Landois)

\section{Conclusion}

L'exemple du plateau de Messigny a été choisi pour le nombre et la diversité des sites archéologiques qui s'y trouvent. Il n'est donc pas représentatif de l'ensemble du Val Suzon, mais les observations faites sur les différents types de structures et les réflexions scientifiques qu'elles soulèvent, peuvent parfaitement être transposées à d'autres secteurs. En effet, on retrouve presque partout dans les forêts du Val Suzon des vestiges d'époques et de natures différentes qui cohabitent aujourd'hui dans un seul et même contexte forestier. Il est néanmoins difficile de parler d'une véritable accumulation de traces d'activités humaines sur un même espace, puisqu'il apparaît bien souvent que les indices archéologiques les plus anciens tendent à s'effacer sous l'effet des occupations successives. Si les quelques sites néolithiques et protohistoriques du Val Suzon attestent bien d'une occupation, de nombreuses incertitudes concernant son organisation à l'échelle du territoire perdurent tout de même. À l'inverse, on commence véritablement à percevoir un paysage antique grâce aux parcellaires et aux fermes présentes sur les plateaux calcaires. Il convient de rester vigilant cependant : ce parcellaire relevé sur le LiDAR n'est peut-être qu'un instantané du découpage de l'espace, qui plus est dans son état final. Ce parcellaire est potentiellement le résultat d'une histoire complexe, ponctuée de réaménagements, où certaines limites ont perduré alors que d'autres ont disparu. Cette remarque vaut également pour les structures médiévales et modernes, même si les archives apportent un éclairage supplémentaire. L'étude des parcellaires découverts dans le Val Suzon s’intéresse aux dynamiques sur le temps long ainsi qu'aux causes de l'abandon de ces terroirs (Landois et al., 2019b). De plus, les relations entre les différentes structures mises au jour dans les parcelles du Grand Continent et de la Mansenne ont d'abord été établies grâce à une analyse minutieuse des données LiDAR et une vérification sur le terrain. Ces observations ont permis d'établir une chronologie des différentes phases d'exploitation de cet espace, entrecoupées d'épisodes de désertion et de reboisement naturel. C'est l'étude des documents d'archives textuels et planimétriques qui est ensuite venue compléter cette datation relative des activités humaines sur le plateau de Messigny. De manière générale, la combinaison et la confrontation des sources profitent considérablement à l'étude du patrimoine historique et archéologique du Val Suzon. 
D'autre part, le plateau de Messigny présente des caractéristiques géomorphologiques particulières qui ont certainement contribué à la préservation de reliefs que l'on pourrait qualifier de « discrets », car il n'est possible de les cartographier qu'à partir des données LiDAR. Un autre facteur participe également à la conservation de ces traces : il s'agit du retour d'un couvert forestier après l'abandon des terres exploitées. Il est pour l'instant assez difficile d'estimer quelles sont les conditions nécessaires pour que ce changement d'environnement soit effectif. Par exemple, faut-il nécessairement une forêt pour protéger les structures, ou bien des friches ou des pelouses sont-elles déjà suffisantes ? Est-ce que cela implique un abandon total de la zone, ou bien certaines activités humaines sont-elles compatibles avec une préservation durable des vestiges ? Pour essayer de répondre à ces questions de préservation, on comprend dès lors qu'il est nécessaire d'étudier l'histoire du territoire et notamment de la forêt. Par ailleurs, il est possible de construire, grâce aux données LiDAR, une carte de l'ancienneté des forêts dans le Val Suzon, suivant la datation des dernières traces d'occupation identifiées pour un endroit donné. Cet âge peut être comparé à la date de la première mention de bois retrouvée dans les archives textuelles. Il apparaît que le massif du Val Suzon ne constitue pas un ensemble uniforme d'un point de vue historique. Ces différences d'ancienneté impliquent potentiellement des disparités environnementales, qui ne s'expliquent donc pas seulement par des conditions naturelles. On suspecte par exemple que certaines activités humaines préexistant à l'installation de la forêt ont modifié les caractéristiques physico-chimiques des sols (Dupouey et al., 2002a ; Dupouey et al., 2002b ; Decocq, 2012; Dambrine et al., 2007). Ainsi, les pratiques agro-pastorales qui ont pris place sur certains plateaux du Val Suzon sur de longues durées ont pu modifier la stabilité des sols et par conséquent les processus d'érosion les affectant (Landois et al., 2020). De plus, l'homme a pu modifier volontairement l'environnement forestier, en sélectionnant par exemple certaines essences. Il faut alors s'intéresser à l'exploitation des ressources sylvicoles, telle qu'elle est décrite dans les archives et à travers les traces matérielles qu'elle a laissées ${ }^{18}$. Le LiDAR s'avère ainsi être un outil parfaitement adapté pour le recensement des activités humaines passées en forêt - si tant est qu'elles aient laissé des traces topographiques - et par conséquent il est aussi un outil opportun pour l'écologie historique (Decocq, 2018).

\section{Bibliographie}

Beck, P., F. Faucher, J.-L. Maigrot (dir.) 2018. Élevage et forêt sur la Montagne dijonnaise à la fin du Moyen Âge : deux établissements forestiers d'éleveurs en Terre de Saint-Seine (Saint-Martin-du-Mont, Côte-d'Or), Drémil-Lafage, Éditions Monique Mergoil, 284 pages.

Bergès, L., J.-L. Dupouey 2017. «Écologie historique et ancienneté de l'état boisé : concepts, avancées et perspectives de la recherche », Revue Forestière Française, 69, 4 : 297-318.

Brulard, R. 1909. «Tumulus de la Mansenne, commune de Messigny (Age du Bronze). », Revue préhistorique illustrée de l'Est de la France, 4 (juillet-aout) : 114-117.

Commission des Antiquités du département de la Côte-d'Or, 1939. Mémoires de la Commission des antiquités du département de la Côte-d’Or (T. 21 1938-1939), Dijon, Imprimerie Bernigaud et Privat, 521 pages.

Chevigny, E., L. Granjon, L. Saligny, D. Goguey, Y. Pautrat, A. Cordier, M. Delcamp 2015. "Detection and identification of archaeological features using aerial LIDAR data in a forested environment (Châtillon-sur-Seine, Côte-d'Or, France)" [Poster], TRAIL 2014 : Formation et recherche pour l'interprétation archéologique des données LiDAR., 2628 mars 2014, Frasne, France, URL : https://hal.archives-ouvertes.fr/hal-01959838 (consulté le 30 juin 2019).

Chevigny E., L. Saligny, L. Granjon, D. Goguey, A. Cordier, Y. Pautrat, A. Giosa 2019. «Identifier et enregistrer les vestiges archéologiques sous couvert forestier à partir des données LiDAR: méthode et limite », Revue d'Archéométrie, 42-2 : 31-43.

\footnotetext{
${ }^{18}$ On pense notamment ici au cas des charbonnières, dont l'étude montre que la production de charbon était omniprésente dans le Val Suzon (Landois et al., 2017). Au vu des volumes et du rythme des coupes, on pourrait même qualifier cette exploitation d'intensive.
} 
Chiffre, J. 1982. «Granges et villages nouveaux en Bourgogne aux XVI ${ }^{\mathrm{e}}$ et XVII ${ }^{\mathrm{e}}$ siècles. Le rôle des abbayes dans la transformation du paysage rural », Revue géographique de l’Est, 22, 3-4 : 183-197.

Cousins, S. A. O. 2001. "Analysis of land-cover transitions based on $17^{\text {th }}$ and $18^{\text {th }}$ century cadastral maps and aerial photograph", Landscape Ecology, $16: 41-54$.

Cremer, G., Poullet, C. 1873. La Campagne de l'Est et l'armée de Bourbaki, Paris, Librairie des célébrités contemporaines, 360 pages.

Dambrine, E., J.-L. Dupouey, L. Laüt, L. Humbert, M. Thinon, T. Beaufils, H. Richard 2007. "Present forest biodiversity patterns in France related to former roman agriculture", Ecology, 88 : 1430-1439.

Decocq, G. 2012 (non publié). «Biodiversité et fertilité selon l'ancienneté de l'état boisé » [Communication à un colloque], 1912-2012, de la statistique Daubrée à l'inventaire forestier de l'IGN : un siècle d'expansion des forêts françaises, 6 décembre 2012, Saint-Mandé, URL : http://www2.agroparistech.fr/podcast/Biodiversite-et-fertilite-selonl-anciennete-de-l-etat-boise.html (consulté le 30 juin 2019).

Decocq, G. 2018. «Décrypter la mémoire forestière au prisme de l'écologie historique : passé, présent et avenir », Les Nouvelles de l'archéologie, $152: 48-53$.

Doneus, M. 2013. "Openness as visualization technique for interpretative mapping of airborne Lidar derived digital terrain models", Remote Sensing, 5, $12:$ 6427-6442.

Drioton, C. 1910. «Essai de classification des enceintes défensives ou non défensives. Murées et tertres des plateaux calcaires de la Côte-d'Or», Mémoires de la Commission des antiquités du département de la Côte-d'Or, 15 (19061910) : 227-237.

Dupouey, J.-L., D. Sciama, W. Koerner, E. Dambrine, J.-C. Rameau 2002a. «La végétation des forêts anciennes », Revue Forestière Française, $54: 521-532$.

Dupouey, J.-L., E. Dambrine, J.-D. Laffite, C. Moares 2002b. "Irreversible impact of past land use on forest soils and biodiversity”, Ecology, 83 : 2798-2984.

Dupouey, J.-L., J. Bachacou, R. Cosserat, S. Aberdam, D. Vallauri, G. Chappart, M.-A. Corsivierde de Villèle 2007. «Vers la réalisation d'une carte géoréférencée des forêts anciennes de France », Le Monde des Cartes, 191 : 85-98.

Dupouey, J.-L., B. Amiaud, S. Chauchard, L. Bergès, J. Abadie, F. Archaux, C. Avon, R. Bec, M. Bonnevialle, M. Burst, T. Cordonnier, M. Deconchat, G. Decocq, M. Delcourte, M. Fuhr, A. Grel, W. Heintz, P. Janssen, G. Landmann, L. Larrieu, N. Leroy, P. Montpied, C. Panaïotis, B. Renaux, X. Rochel, M. Thomas, A. Salvaudon, D. Vallauri, A. Villemey 2016 (non publié). «Cartographie des forêts anciennes de France : objectifs, bilan et perspectives » [Communication à un colloque], Géohistoire de l'environnement et des paysages, 12-14 octobre 2016, Toulouse, URL : http://oatao.univ-toulouse.fr/17522/ (consulté le 30 juin 2019).

Favory, F. 2012. «Les parcellaires antiques de Gaule médiane et septentrionale », in : Carpentier, V., Marcigny, C. (dir.), Des hommes aux champs. Pour une archéologie des espaces ruraux du Néolithique au Moyen Âge, Rennes, Presses universitaires de Rennes : 111-130.

Georges-Leroy, M., J. Bock, E. Dambrine, J.-L.Dupouey 2009. «Le massif forestier, objet pertinent pour la recherche archéologique : l'exemple forestier de Haye (Meurthe-et-Moselle)», Revue géographique de l'Est, 49 : 2-17.

Georges-Leroy, M., J. Bock, E. Dambrine, J.-L. Dupouey, A. Gebhardt, J.-D. Laffite 2012. «Les vestiges gallo-romains conservés dans le massif forestier de Haye (Meurthe-et-Moselle). Leur apport à l'étude de l'espace agraire », in : V. Carpentier, C. Marcigny (dir.), Des hommes aux champs. Pour une archéologie des espaces ruraux du Néolithique au Moyen Âge, Rennes, Presses Universitaires de Rennes : 157-180.

Georges-Leroy, M., J. Bock, E. Dambrine, J.-L. Dupouey, J.-D. Laffite 2014 (non publié). «Parcellaires et habitat antiques des forêts du plateau de Haye en Lorraine : bilan et perspectives » [Communication à un colloque], Les parcellaires conservés sous forêt, 5 mai 2014, Paris, URL : https://hal.archives-ouvertes.fr/hal-01067845 (consulté le 30 juin 2019).

Georges-Leroy, M. 2015 (non publié). «Typologie des structures agraires et parcellaires fossilisées sous couvert forestier en Lorraine» [Communication à un colloque], LiDAR \& Pratiques, 19-20 mai 2015, Besançon, URL: https://odit.hypotheses.org/telechargements-5/cycle-de-seminaires-lidar-pratiques (consulté le 30 juin 2019).

Goguey, D., Y. Pautrat, J.-P. Guillaumet, J.-P. Thevenot, L. Popovitch 2010. «Dix ans d'archéologie forestière dans le Châtillonnais (Côte-d'Or) : enclos, habitats, parcellaires », Revue Archéologique de l'Est, 59 : 99-209.

Hesse, R. 2010. "LiDAR-derived Local Relief Models: a new tool for archaeological prospection", Archaeological Prospection, $17: 67-72$. 
Landois, R., J.-P. Garcia, A. Quiquerez 2017. "Spatial analysis of charcoal kilns in Val Suzon (Burgundy, France): quantitative and geographical approach of wood charcoal economy" [Poster] International workshop of pedoanthracology: the memory of the soils and the wood charcoals, 2-3 novembre 2017, Limoges, France, URL : https://hal.archives-ouvertes.fr/hal-02190581 (consulté le 30 juin 2019).

Landois, R., J.-P. Garcia, A. Quiquerez 2019a. «Un site d'élevage de lapins de la fin du Moyen Âge : les garennes de Sainte-Foy (Val-Suzon, Côte-d'Or)», Revue Archéologique de l'Est, 68 : 285-298.

Landois, R., J.-P. Garcia, A. Quiquerez 2019b. «Un habitat groupé antérieur au XII ${ }^{\mathrm{e}}$ siècle préservé sous forêt : le site de Goa (Val-Suzon, Côte-d'Or)», Bulletin du Centre d'Études Médiévales d'Auxerre, 23, 1, URL : https://journals.openedition.org/cem/16385 (consulté le 16 décembre 2019).

Landois, R., J.-P. Garcia, A. Quiquerez 2020 (à paraitre). «Enregistrement des activités humaines et impacts sur les transferts sédimentaires en contexte de plateaux/vallées calcaires: la forêt du Val Suzon sur le temps long» [Communication à un colloque], 26 ${ }^{\mathrm{e}}$ Réunion des Sciences de la Terre, 22-26 octobre 2018, Lille, URL: https://hal.archives-ouvertes.fr/hal-01937508 (consulté le 30 juin 2019).

Ledeuil d’Enquin, J. 1893 - Le général Bosak, comte de Hauké : armée des Vosges. Paris, Librairie militaire E. Dubois, 73 pages.

de Matos Machado, R., J.-P. Amat, G. Arnaud-Fassetta, F. Bétard 2016. «Potentialités de l'outil LiDAR pour cartographier des vestiges de la Grande Guerre en milieu intra-forestier (bois des Caures, forêt domaniale de Verdun, Meuse) » EchoGéo, 38, URL : http://journals.openedition.org/echogeo/14791 (consulté le 30 juin 2019).

Nicolardot, J.-P. 2003. L'habitat fortifié pré- et protohistorique en Côte-d'Or : les camps de Myard à Vitteaux et du Châtelet d'Étaules dans le contexte archéologique régional ( $\mathrm{du} \mathrm{V}^{\mathrm{e}}$ millénaire au IV $\mathrm{V}^{\mathrm{e}}$ siècle avant J.-C.). Dijon, Revue Archéologique de l'Est, 374 pages.

Office National des Forêts, 2016. Le Val Suzon. Dossier de candidature au label Forêt d'Exception. Dijon, Office National des Forêts, 68 pages.

Paradis-Grenouillet, S., S. Burri, R. Rouaud (dir.) 2018. Charbonnage, charbonniers, charbonnières : confluence de regards autour d'un artisanat méconnu, Aix-en-Provence, Presses universitaires de Provence, 238 pages.

Pautrat, Y., A. Charmot, 2013 (non publié). « Nécropoles tumulaires versus épierrement agricole », 24 pages.

Provost, M. (dir.) 2009. Carte archéologique de la Gaule : la Côte-d'Or (T. 2 d'Allerey à Normier), Paris, Académie des inscriptions et belles-lettres, 651 pages.

Rassat, G., R. Crouzevialle, F. Cerbelaud, P. Allée, M.-C. Bal-Serin 2018. «L'utilisation et l'apport des données LiDAR pour l'étude des forêts en Charente», in: S. Paradis-Grenouillet, S. Burri, R. Rouaud (dir.) 2018. Charbonnage, charbonniers, charbonnières : confluence de regards autour d'un artisanat méconnu, Aix-en-Provence, Presses universitaires de Provence : 105-120.

Saint Jacob, P. (de) 1995. Les paysans de la Bourgogne du Nord au dernier siècle de l'Ancien Régime. Rennes/Dijon, Association d'histoire des sociétés rurales/Éditions universitaires de Dijon, 643 pages.

Schwartz, D., D. Ertlen, J. Battmann, M. Caspard, A. Gebhart, S. Goepp, F. Basoge, L. Koupaliantz, B. Metz 2012. «Études actuelles sur un type de paysage encore très peu connu en Alsace : les paysages d'enclos médiévaux. Extension, typologie, éléments de datation », in : V. Carpentier, C. Marcigny (dir.), Des hommes aux champs. Pour une archéologie des espaces ruraux du Néolithique au Moyen Âge, Rennes, Presses universitaires de Rennes : $305-$ 326.

Sittler, B. 2015 (non publié). «Les apports du LiDAR pour appréhender les modèles agraires : l'exemple du Fossé Rhénan» [Communication à un colloque], LiDAR \& Pratiques, 19-20 mai 2015, Besançon, URL: https://odit.hypotheses.org/telechargements-5/cycle-de-seminaires-lidar-pratiques (consulté le 30 juin 2019).

Sittler, B., K. Hauger 2007. «Les apports du laser aéroporté à la documentation de parcellaires anciens fossilisés par la forêt : l'exemple des champs bombés de Rastatt en Pays de Bade», in : J.-L. Dupouey, E. Dambrine, C. Dardignac, M. Georges-Leroy (éd.), La mémoire des forêts. Actes du colloque «Forêt, archéologie et environnement» 14-16 décembre 2004, Paris/Metz, Office national des Forêts/Institut national de la recherche agronomique/Direction régionale des affaires culturelles de Lorraine : 155-161.

Vallauri, D., A. Grel, E. Granier, J.-L. Dupouey 2012. Les forêts de Cassini : analyse quantitative et comparaison avec les forêts actuelles, Marseille, World Wildlife Fund/Institut national de la recherche agronomique, 64 pages.

Kokalj, Z., M. Somrak 2019. "Why Not a Single Image? Combining Visualizations to Facilitate Fieldwork and On-Screen Mapping", Remote Sensing, 11, $7: 747$. 
Kokalj, Z., K. Zaksek, K. Ostir 2011. "Application of sky-view factor for the visualisation of historic landscape features in lidar-derived relief models", Antiquity, 85 : 263-273. 